\title{
Stability and Hopf Bifurcation in a Delayed HIV Infection Model with General Incidence Rate and Immune Impairment
}

\author{
Fuxiang Li, ${ }^{1}$ Wanbiao Ma, ${ }^{1}$ Zhichao Jiang, ${ }^{2}$ and Dan $\mathrm{Li}^{1}$ \\ ${ }^{1}$ Department of Applied Mathematics, School of Mathematics and Physics, University of Science and Technology Beijing, \\ Beijing 100083, China \\ ${ }^{2}$ Fundamental Science Department, North China Institute of Aerospace Engineering, Langfang, Hebei 065000, China
}

Correspondence should be addressed to Wanbiao Ma; wanbiao_ma@ustb.edu.cn

Received 16 May 2015; Accepted 29 June 2015

Academic Editor: Chung-Min Liao

Copyright (C) 2015 Fuxiang Li et al. This is an open access article distributed under the Creative Commons Attribution License, which permits unrestricted use, distribution, and reproduction in any medium, provided the original work is properly cited.

\begin{abstract}
We investigate the dynamical behavior of a delayed HIV infection model with general incidence rate and immune impairment. We derive two threshold parameters, the basic reproduction number $R_{0}$ and the immune response reproduction number $R_{1}$. By using Lyapunov functional and LaSalle invariance principle, we prove the global stability of the infection-free equilibrium and the infected equilibrium without immunity. Furthermore, the existence of Hopf bifurcations at the infected equilibrium with CTL response is also studied. By theoretical analysis and numerical simulations, the effect of the immune impairment rate on the stability of the infected equilibrium with CTL response has been studied.
\end{abstract}

\section{Introduction}

In recent years, mathematical models have been proved to be valuable in understanding the dynamics of viral infection (see, e.g., [1-8]). In most virus infections, cytotoxic T lymphocyte (CTL) cells play a significant role in antiviral defense by attacking virus-infected cells. In order to study the role of the population dynamics of the viral infection with CTL response, Nowak and Bangham et al. proposed a basic viral infection model describing the interactions between a replicating virus population and a specific antiviral CTL response, which takes into account four populations: uninfected cells, actively infected cells, free virus, and CTL cells (see, e.g., $[1-4,9,10])$. Now, the population dynamics of viral infection with CTL response has been paid much attention and many properties have been investigated (see, e.g., [11-16]).

Furthermore, the state of latent infection cannot be ignored in many biological models. The infected cells are separated into two distinct compartments, latently infected and actively infected. These latently infected cells do not produce virus and can evade from viral cytopathic effects and host immune mechanisms (see, e.g., [17-20]). Recently, the following model with latent infection and CTL response has been proposed (see, e.g., [11]):

$$
\begin{aligned}
& \dot{x}(t)=\lambda-\beta x(t) v(t)-\mu_{1} x(t), \\
& \dot{u}(t)=\beta x(t) v(t)-\left(\sigma+\mu_{2}\right) u(t), \\
& \dot{y}(t)=\sigma u(t)-p y(t) z(t)-\mu_{3} y(t), \\
& \dot{v}(t)=k y(t)-\mu_{4} v(t), \\
& \dot{z}(t)=q y(t) z(t)-\mu_{5} z(t),
\end{aligned}
$$

where $x(t), u(t), y(t), v(t)$, and $z(t)$ represent the numbers of uninfected cells, latently infected cells, actively infected cells, free virus, and CTLs at time $t$, respectively. Uninfected cells are produced at the rate $\lambda$, die at the rate $\mu_{1}$, and become infected at the rate $\beta$. The constant $\sigma$ is the rate of latently infected cells translating to actively infected cells and $\mu_{3}$ is the death rate of actively infected cells. The constant $\mu_{2}$ represents the death rate of latently infected cells. The constant $p$ is the rate of CTL-mediated lysis and $q$ is the rate of CTL proliferation. The constant $k$ is the rate of production of virus by infected cells and $\mu_{4}$ is the clearance rate of free virus. The removal rate of CTLs is $\mu_{5}$. 
However, in plenty of previous papers, many models are constructed under the assumption that the presence of antigen can stimulate immunity and ignore the immune impairment (see, e.g., $[8,11,16,17])$. In fact, some pathogens can also suppress immune response or even destroy immunity especially when the load of pathogens is too high such as HIV, HBV (see, e.g., [15, 21-25]). Regoes et al. consider an ordinary differential equation (ODE) model with an immune impairment term $m y z$ (see, e.g., $[12,26,27]$ ), where $m$ denotes the immune impairment rate. Time delay should be considered in models for CTL response. It is shown that time delay plays an important role to the dynamic properties in models for CTL response (see, e.g., $[1,5,6,8,15]$ ). In fact, antigenic stimulation generating CTLs may need a period of time $t$; that is, the CTL response at time $t$ may depend on the numbers of CTLs and infected cells at time $t-\tau$, for a time $\operatorname{lag} \tau>0$ (see, e.g., $[1,5,13]$ ).

Motivated by the above works, in this paper, we will study a delay differential equation (DDE) model of HIV infection with immune impairment and delayed CTL response. Furthermore, we know that the actual incidence rate is probably not linear over the entire range of $x$ and $v$. Based on the works mentioned above (see, e.g., [21, 28-31]), we propose the following system with general incidence function:

$$
\begin{aligned}
& \dot{x}(t)=\lambda-f(x(t), v(t)) v(t)-\mu_{1} x(t), \\
& \dot{u}(t)=f(x(t), v(t)) v(t)-\left(\sigma+\mu_{2}\right) u(t), \\
& \dot{y}(t)=\sigma u(t)-p y(t) z(t)-\mu_{3} y(t), \\
& \dot{v}(t)=k y(t)-\mu_{4} v(t), \\
& \dot{z}(t)=q y(t-\tau) z(t-\tau)-\mu_{5} z(t)-m y(t) z(t),
\end{aligned}
$$

where the state variables $x(t), u(t), y(t), v(t)$, and $z(t)$ and the parameters $\lambda, \sigma, p, k, q, \mu_{1}, \mu_{2}, \mu_{3}, \mu_{4}$, and $\mu_{5}$ have the same biological meaning as in system (1). $m$ is the immune impairment rate. Suppose all the parameters are nonnegative. We assume the incidence rate is the general incidence function $f(x, v) v$, where $f \in C^{1}([0,+\infty] \times$ $[0,+\infty], R)$ satisfies the following hypotheses:

(H1) $f(x, v) v \geq 0$, for all $x \geq 0$ and $v \geq 0 ; f(x, v)=0$ if and only if $x=0$;

(H2) $\partial f(x, v) / \partial x>0$, for all $x \geq 0$ and $v \geq 0$;

(H3) $\partial f(x, v) / \partial v \leq 0$, for all $x \geq 0$ and $v \geq 0$;

(H4) $\partial(f(x, v) v) / \partial v>0$, for all $x>0$ and $v \geq 0$.

Clearly, the hypotheses can be satisfied by different types of the incidence rate including the mass action, the Holling type II function, the saturation incidence, BeddingtonDeAngelis incidence function, Crowley-Martin incidence function, and the more generalized incidence functions (see, e.g., $[4,6,17,32,33])$. Further, in order to study the global stability of the equilibria of system (2) by the method of Lyapunov functionals, we assume the following hypotheses hold (see, e.g., [28]):

(H5) $x-x_{0}-\int_{x_{0}}^{x}\left(f\left(x_{0}, 0\right) / f(s, 0)\right) d s \rightarrow+\infty$, as $x \rightarrow+\infty$ or $x \rightarrow 0^{+}$;

$$
\begin{aligned}
& \text { (H6) } x-x_{1}-\int_{x_{1}}^{x}\left(f\left(x_{1}, v_{1}\right) / f\left(s, v_{1}\right)\right) d s \rightarrow+\infty \text {, as } x \rightarrow \\
& \quad+\infty \text { or } x \rightarrow 0^{+} ; \\
& \text {(H7) } x-x^{*}-\int_{x^{*}}^{x}\left(f\left(x^{*}, v^{*}\right) / f\left(s, v^{*}\right)\right) d s \rightarrow+\infty \text {, as } x \rightarrow \\
& \quad+\infty \text { or } x \rightarrow 0^{+} .
\end{aligned}
$$

The main purpose of this paper is to carry out a complete theoretical analysis on the global stability of the equilibria of system (2). The organization of this paper is as follows. In Section 2, we consider the nonnegativity and boundedness of the solutions and the existence of the equilibria of system (2). In Section 3, we consider the global stability of the infectionfree equilibrium $E_{0}$ and the infected equilibrium without immunity $E_{1}$ by constructing suitable Lyapunov functionals and using LaSalle invariance principle. In Section 4 , we discuss the local stability of the infected equilibrium with CTL response $E^{*}$ and the existence of Hopf bifurcations. Finally, in Section 5, the brief conclusions are given and some numerical simulations are carried out to illustrate the main results.

\section{Basic Results}

2.1. The Nonnegativity and Boundedness of the Solutions. According to biological meanings, the initial condition of system (2) is given as follows:

$$
\begin{array}{ll}
x(\theta)=\varphi_{1}(\theta), & u(\theta)=\varphi_{2}(\theta), \quad y(\theta)=\varphi_{3}(\theta), \\
v(\theta)=\varphi_{4}(\theta), & z(\theta)=\varphi_{5}(\theta),
\end{array}
$$

where $\theta \in[-\tau, 0]$ and $\left(\varphi_{1}, \varphi_{2}, \varphi_{3}, \varphi_{4}, \varphi_{5}\right) \in C=C\left([-\tau, 0], R_{+}^{5}\right)$ and $C$ is the Banach space of the continuous functions mapping the interval $[-\tau, 0]$ into $R_{+}^{5}, R_{+}^{5}=\left\{\left(x_{1}, x_{2}, x_{3}, x_{4}, x_{5}\right) \mid\right.$ $\left.x_{i} \geq 0, i=1,2,3,4,5\right\}$.

Under the initial condition (3), it easily shows that the solution of system (2) is unique and nonnegative for all $t \geq 0$ and ultimately bounded. It has the following result.

Proposition 1. Under the initial condition (3), the solution of system (2) is unique and nonnegative for all $t \geq 0$ and also ultimately bounded, when (H1)-(H7) are satisfied.

Proof. The uniqueness and nonnegativity of the solution $(x(t), u(t), y(t), v(t), z(t))$ can be easily proved by using the theorems in $[34,35]$.

Next, for $t \geq 0$, define

$$
L(t)=x(t)+u(t)+y(t)+\frac{\mu_{3}}{2 k} v(t)+\frac{p}{2 q} z(t+\tau) .
$$

By the nonnegativity of the solutions, it follows that, for $t \geq 0$,

$$
\begin{aligned}
L^{\prime}(t)= & \lambda-\mu_{1} x(t)-\mu_{2} u(t)-\frac{\mu_{3}}{2} y(t)-\frac{\mu_{3} \mu_{4}}{2 k} v(t) \\
& -\frac{p \mu_{5}}{2 q} z(t+\tau)-\frac{p}{2} y(t) z(t) \\
& -\frac{p m}{2 q} y(t+\tau) z(t+\tau) \leq \lambda-\gamma L(t),
\end{aligned}
$$


where $\gamma=\min \left\{\mu_{1}, \mu_{2}, \mu_{3} / 2, \mu_{4}, \mu_{5}\right\}$. Thus, it has that $\limsup _{t \rightarrow+\infty} L(t) \leq \lambda / \gamma$, from which it has that the solution $(x(t), u(t), y(t), v(t), z(t))$ is ultimately bounded.

2.2. The Existence of the Equilibria. Next, we consider the existence of the equilibria. The equilibrium of system (2) satisfies

$$
\begin{array}{r}
\lambda-f(x, v) v-\mu_{1} x=0, \\
f(x, v) v-\left(\sigma+\mu_{2}\right) u=0, \\
\sigma u-p y z-\mu_{3} y=0, \\
k y-\mu_{4} v=0, \\
q y z-\mu_{5} z-m y z=0 .
\end{array}
$$

If $u=0, y=0, v=0$, and $z=0$, system (2) has only one equilibrium, that is, the infection-free equilibrium $E_{0}=$ $\left(x_{0}, 0,0,0,0\right)$, where $x_{0}=\lambda / \mu_{1}$.

If $u \neq 0, y \neq 0, v \neq 0$, and $z=0$, we have

$$
\begin{aligned}
& f\left(x, \frac{k \sigma\left(\lambda-\mu_{1} x\right)}{\mu_{3} \mu_{4}\left(\sigma+\mu_{2}\right)}\right)-\frac{\mu_{3} \mu_{4}\left(\sigma+\mu_{2}\right)}{k \sigma}=0, \\
& y=\frac{\sigma\left(\lambda-\mu_{1} x\right)}{\mu_{3}\left(\sigma+\mu_{2}\right)}, \\
& u=\frac{\lambda-\mu_{1} x}{\sigma+\mu_{2}} \\
& v=\frac{k \sigma\left(\lambda-\mu_{1} x\right)}{\mu_{3} \mu_{4}\left(\sigma+\mu_{2}\right)} .
\end{aligned}
$$

Since $v>0$, we have that $x<\lambda / \mu_{1}$. Hence, we only need to consider the case of $x<\lambda / \mu_{1}$.

Consider the following function defined on the interval $\left(0, \lambda / \mu_{1}\right)$ by

$$
F(x)=f\left(x, \frac{k \sigma\left(\lambda-\mu_{1} x\right)}{\mu_{3} \mu_{4}\left(\sigma+\mu_{2}\right)}\right)-\frac{\mu_{3} \mu_{4}\left(\sigma+\mu_{2}\right)}{k \sigma} .
$$

Under hypotheses (H2) and (H3), we have

$$
F^{\prime}(x)=\frac{\partial f}{\partial x}+\frac{\partial f}{\partial v}\left(\frac{-k \sigma \mu_{1}}{\mu_{3} \mu_{4}\left(\sigma+\mu_{2}\right)}\right)>0 .
$$

We know that the function $F(x)$ is strictly monotonically increasing with respect to $x$. Denote the basic reproduction number $R_{0}$ of system (2) by

$$
R_{0}=\frac{k \sigma f\left(\lambda / \mu_{1}, 0\right)}{\mu_{3} \mu_{4}\left(\sigma+\mu_{2}\right)} .
$$

Clearly, we have

$$
\begin{aligned}
F(0) & =-\frac{\mu_{3} \mu_{4}\left(\sigma+\mu_{2}\right)}{k \sigma}<0, \\
F\left(\frac{\lambda}{\mu_{1}}\right) & =f\left(\frac{\lambda}{\mu_{1}}, 0\right)-\frac{\mu_{3} \mu_{4}\left(\sigma+\mu_{2}\right)}{k \sigma} \\
& =\frac{\mu_{3} \mu_{4}\left(\sigma+\mu_{2}\right)}{k \sigma}\left(R_{0}-1\right) .
\end{aligned}
$$

It has that there exists a unique $x_{1} \in\left(0, \lambda / \mu_{1}\right)$ such that $F\left(x_{1}\right)=0$, if $R_{0}>1$. Then we can compute $u_{1}, y_{1}$ and $v_{1}$ by (8). Hence, we get the unique infected equilibrium without immunity $E_{1}=\left(x_{1}, u_{1}, y_{1}, v_{1}, 0\right)$.

If $z \neq 0$ and $q>m$, we get the following equations:

$$
\begin{aligned}
& f\left(x, \frac{k \mu_{5}}{\mu_{4}(q-m)}\right) \frac{k \mu_{5}}{\mu_{4}(q-m)}-\lambda+\mu_{1} x=0, \\
& u=\frac{\lambda-\mu_{1} x}{\sigma+\mu_{2}} \\
& y=\frac{\mu_{5}}{q-m}>0, \\
& v=\frac{k \mu_{5}}{\mu_{4}(q-m)}>0, \\
& z=\frac{\left(\lambda-\mu_{1} x\right)(q-m) \sigma-\mu_{3} \mu_{5}\left(\sigma+\mu_{2}\right)}{p \mu_{5}\left(\sigma+\mu_{2}\right)} .
\end{aligned}
$$

Since $z>0$, we have $x<\bar{x}$, where

$$
\bar{x}=\frac{\lambda(q-m) \sigma-\mu_{3} \mu_{5}\left(\sigma+\mu_{2}\right)}{\mu_{1}(q-m) \sigma} .
$$

Hence, the existence of the equilibrium requires $\bar{x}>0$ and (13) has a solution on the interval $(0, \bar{x})$.

Denote

$$
\bar{R}=\frac{\lambda(q-m) \sigma}{\mu_{3} \mu_{5}\left(\sigma+\mu_{2}\right)}
$$

Hence, if $\bar{R}>1$, it has $\bar{x}>0$. Denote

$$
G(x)=f\left(x, \frac{k \mu_{5}}{\mu_{4}(q-m)}\right) \frac{k \mu_{5}}{\mu_{4}(q-m)}-\lambda+\mu_{1} x .
$$

Under hypothesis (H2), we know that the function $G(x)$ is strictly monotonically increasing with respect to $x$. Clearly, we have

$$
\begin{aligned}
G(0)= & -\lambda<0, \\
G(\bar{x})= & f\left(\bar{x}, \frac{k \mu_{5}}{\mu_{4}(q-m)}\right) \frac{k \mu_{5}}{\mu_{4}(q-m)}-\lambda+\mu_{1} \bar{x} \\
= & f\left(\bar{x}, \frac{k \mu_{5}}{\mu_{4}(q-m)}\right) \frac{k \mu_{5}}{\mu_{4}(q-m)} \\
& -\frac{\mu_{3} \mu_{5}\left(\sigma+\mu_{2}\right)}{(q-m) \sigma}=\frac{\mu_{3} \mu_{5}\left(\sigma+\mu_{2}\right)}{(q-m) \sigma}\left(R_{1}-1\right),
\end{aligned}
$$

where

$$
R_{1}=\frac{k \sigma f\left(\bar{x}, k \mu_{5} / \mu_{4}(q-m)\right)}{\mu_{3} \mu_{4}\left(\sigma+\mu_{2}\right)} .
$$

Hence, we have that there exists $x^{*} \in(0, \bar{x})$ such that $G\left(x^{*}\right)=$ 0 , if $\bar{R}>1$ and $R_{1}>1$. Then we can compute $u^{*}, y^{*}, v^{*}$, and $z^{*}$ by (14) and (15). 
Denote the immune response reproduction number of system (2) as $R_{1}$. Therefore, we have that there exists a unique infected equilibrium with CTL response $E^{*}=\left(x^{*}, u^{*}, y^{*}\right.$, $\left.v^{*}, z^{*}\right)$, if $\bar{R}>1$ and $R_{1}>1$. This proves the following theorem.

Theorem 2. Suppose that hypotheses (H1)-(H4) are satisfied; the following conclusions hold.

(i) System (2) always has an infection-free equilibrium $E_{0}$.

(ii) System (2) has an infected equilibrium without immunity $E_{1}$ if $R_{0}>1$.

(iii) System (2) has an infected equilibrium with immunity $E^{*}$ if $\bar{R}>1$ and $R_{1}>1$.

From hypotheses (H1)-(H3), it is clear that $R_{1}<R_{0}$. In order to study the global stability of the infected equilibrium $E_{1}$ in the next section, we give the following remark.

Remark 3. Suppose that $\bar{R}>1$ is satisfied; then the following results hold:

(i) If $R_{1}>1$, then $(q-m) y_{1} / \mu_{5}>1$.

(ii) If $R_{1} \leq 1$, then $(q-m) y_{1} / \mu_{5} \leq 1$.

Let us give the proof of Remark 3. Firstly, for Case (i), since $R_{1}>1$, then

$$
\begin{aligned}
F(\bar{x}) & =f\left(\bar{x}, \frac{k \sigma\left(\lambda-\mu_{1} \bar{x}\right)}{\mu_{3} \mu_{4}\left(\sigma+\mu_{2}\right)}\right)-\frac{\mu_{3} \mu_{4}\left(\sigma+\mu_{2}\right)}{k \sigma} \\
& =\frac{\mu_{3} \mu_{4}\left(\sigma+\mu_{2}\right)}{k \sigma}\left(R_{1}-1\right)>0 .
\end{aligned}
$$

Since the function $F(x)$ is strictly monotonically increasing with respect to $x$ and $F\left(x_{1}\right)=0$, we have $x_{1}<\bar{x}$. Therefore

$$
\lambda-\mu_{1} x_{1}>\lambda-\mu_{1} \bar{x}=\frac{\mu_{3} \mu_{5}\left(\sigma+\mu_{2}\right)}{\sigma(q-m)} .
$$

Then

$$
\frac{(q-m) y_{1}}{\mu_{5}}=\frac{q-m}{\mu_{5}} \cdot \frac{\sigma\left(\lambda-\mu_{1} x_{1}\right)}{\mu_{3}\left(\sigma+\mu_{2}\right)}>1 .
$$

Secondly, for Case (ii), since $R_{1} \leq 1$, then

$$
\begin{aligned}
F(\bar{x}) & =f\left(\bar{x}, \frac{k \sigma\left(\lambda-\mu_{1} \bar{x}\right)}{\mu_{3} \mu_{4}\left(\sigma+\mu_{2}\right)}\right)-\frac{\mu_{3} \mu_{4}\left(\sigma+\mu_{2}\right)}{k \sigma} \\
& =\frac{\mu_{3} \mu_{4}\left(\sigma+\mu_{2}\right)}{k \sigma}\left(R_{1}-1\right) \leq 0 .
\end{aligned}
$$

We have $x_{1} \geq \bar{x}$. Therefore

$$
\lambda-\mu_{1} x_{1} \leq \lambda-\mu_{1} \bar{x}=\frac{\mu_{3} \mu_{5}\left(\sigma+\mu_{2}\right)}{\sigma(q-m)} .
$$

Then

$$
\frac{(q-m) y_{1}}{\mu_{5}}=\frac{q-m}{\mu_{5}} \cdot \frac{\sigma\left(\lambda-\mu_{1} x_{1}\right)}{\mu_{3}\left(\sigma+\mu_{2}\right)} \leq 1 .
$$

\section{The Global Stability of the Equilibria}

In this section, we study the global stability of the equilibria of system (2). Firstly, we analyze the global stability of the infection-free equilibrium $E_{0}$.

Theorem 4. Suppose that hypotheses (H1)-(H7) are satisfied. If $R_{0} \leq 1$, then the infection-free equilibrium $E_{0}$ is globally asymptotically stable for any time delay $\tau \geq 0$. If $R_{0}>1$, then the infection-free equilibrium $E_{0}$ is unstable for any time delay $\tau \geq 0$.

Proof. Let $(x(t), u(t), y(t), v(t), z(t))$ be a positive solution of system (2) with the initial condition (3) for $t \geq 0$. Motivated by the works in $[14,28,31,36,37]$, we consider the following Lyapunov functional:

$$
\begin{aligned}
V_{1}= & x-x_{0}-\int_{x_{0}}^{x} \frac{f\left(x_{0}, 0\right)}{f(s, 0)} d s+u+\frac{\sigma+\mu_{2}}{\sigma} y \\
& +\frac{\mu_{3}\left(\sigma+\mu_{2}\right)}{k \sigma} v+\frac{\sigma+\mu_{2}}{\sigma} \frac{p}{q-m} z \\
& +\frac{\sigma+\mu_{2}}{\sigma} \frac{p}{q-m} \int_{t-\tau}^{t} q y(\theta) z(\theta) d \theta,
\end{aligned}
$$

where $\lambda=\mu_{1} x_{0}$. By (H1)-(H5), it is obvious that $V_{1}$ is positive definite with respect to $E_{0}$. For $t \geq 0$, the time derivative of $V_{1}$ along the solutions of system (2) is

$$
\begin{aligned}
\dot{V}_{1} & \left(1-\frac{f\left(x_{0}, 0\right)}{f(x, 0)}\right) \dot{x}+\dot{u}+\frac{\sigma+\mu_{2}}{\sigma} \dot{y}+\frac{\mu_{3}\left(\sigma+\mu_{2}\right)}{k \sigma} \dot{v} \\
& +\frac{\sigma+\mu_{2}}{\sigma} \frac{p}{q-m} \dot{z} \\
& +\frac{\sigma+\mu_{2}}{\sigma} \frac{p}{q-m}[q y(t) z(t)-q y(t-\tau) z(t-\tau)] \\
= & \mu_{1}\left(1-\frac{f\left(x_{0}, 0\right)}{f(x, 0)}\right)\left(x_{0}-x\right)+\frac{f\left(x_{0}, 0\right)}{f(x, 0)} f(x, v) v \\
& -\frac{\mu_{3} \mu_{4}\left(\sigma+\mu_{2}\right)}{k \sigma} v-\frac{\sigma+\mu_{2}}{\sigma} \frac{p}{q-m} \mu_{5} z \\
= & \mu_{1}\left(1-\frac{f\left(x_{0}, 0\right)}{f(x, 0)}\right)\left(x_{0}-x\right) \\
& -\frac{\mu_{3} \mu_{4}\left(\sigma+\mu_{2}\right)}{k \sigma}\left(1-\frac{f(x, v)}{f(x, 0)} R_{0}\right) v \\
& -\frac{p+\mu_{2}}{q-m} \mu_{5} z .
\end{aligned}
$$

Since hypotheses (H1)-(H3) and $R_{0} \leq 1$, we have

$$
\begin{array}{r}
\mu_{1}\left(1-\frac{f\left(x_{0}, 0\right)}{f(x, 0)}\right)\left(x_{0}-x\right) \leq 0, \\
1-\frac{f(x, v)}{f(x, 0)} R_{0} \geq 0 .
\end{array}
$$


Therefore, $\dot{V}_{1} \leq 0$ if $R_{0} \leq 1$. Then it follows from stability theorems in $[34,35]$ that the infection-free equilibrium $E_{0}$ is stable for any time delay $\tau \geq 0$ if $R_{0} \leq 1$.

Furthermore, note that, for each $t \geq 0, \dot{V}_{1}=0$ implies that $x(t)=x_{0}, z(t)=0$. Let $M$ be the largest invariant set in the set

$$
\begin{aligned}
\Gamma_{1} & =\left\{\left(\varphi_{1}, \varphi_{2}, \varphi_{3}, \varphi_{4}, \varphi_{5}\right) \in C \mid \dot{V}_{1}=0\right\} \\
& \subset\left\{\left(\varphi_{1}, \varphi_{2}, \varphi_{3}, \varphi_{4}, \varphi_{5}\right) \in C \mid \varphi_{1}(0)=x_{0}, \varphi_{5}(0)\right. \\
& =0\} .
\end{aligned}
$$

We have from the first four equations of system (2) and the invariance of $M$ that $M=\left\{E_{0}\right\}$. Since any solution of system (2) is bounded, it follows from LaSalle invariance principle (see, e.g., $[34,35]$ ) that the infection-free equilibrium $E_{0}$ is also globally attractive for any time delay $\tau \geq 0$ if $R_{0} \leq 1$.

The characteristic equation of system (2) at the infectionfree equilibrium $E_{0}$ is

$$
\begin{aligned}
(s & \left.+\mu_{1}\right)\left(s+\mu_{5}\right)\left[s^{3}+\left(\sigma+\mu_{2}+\mu_{3}+\mu_{4}\right) s^{2}\right. \\
& +\left(\left(\mu_{2}+\sigma\right)\left(\mu_{3}+\mu_{4}\right)+\mu_{3} \mu_{4}\right) s \\
& \left.+\mu_{3} \mu_{4}\left(\sigma+\mu_{2}\right)\left(1-R_{0}\right)\right]=0 .
\end{aligned}
$$

Clearly, if $R_{0}>1$, (31) has at least a positive real root. Thus, the infection-free equilibrium $E_{0}$ is unstable.

Next we study the global stability of the infected equilibrium without immunity $E_{1}$.

Theorem 5. Suppose that hypotheses (H1)-(H7) and $\bar{R}>1$ are satisfied. If $R_{0}>1 \geq R_{1}$, then the infected equilibrium without immunity $E_{1}$ is globally asymptotically stable for any time delay $\tau \geq 0$. If $R_{1}>1$, then the infected equilibrium without immunity $E_{1}$ is unstable for any time delay $\tau \geq 0$.

Proof. Let $(x(t), u(t), y(t), v(t), z(t))$ be a positive solution of system (2) with the initial condition (3) for $t \geq 0$. Consider the following Lyapunov functional:

$$
\begin{aligned}
V_{2}= & x-x_{1}-\int_{x_{1}}^{x} \frac{f\left(x_{1}, v_{1}\right)}{f\left(s, v_{1}\right)} d s+\left(u-u_{1}-u_{1} \ln \frac{u}{u_{1}}\right) \\
& +\frac{\sigma+\mu_{2}}{\sigma}\left(y-y_{1}-y_{1} \ln \frac{y}{y_{1}}\right) \\
& +\frac{\mu_{3}\left(\sigma+\mu_{2}\right)}{k \sigma}\left(v-v_{1}-v_{1} \ln \frac{v}{v_{1}}\right) \\
& +\frac{\sigma+\mu_{2}}{\sigma} \frac{p}{q-m} z \\
& +\frac{\sigma+\mu_{2}}{\sigma} \frac{p q}{q-m} \int_{t-\tau}^{t} y(\theta) z(\theta) d \theta .
\end{aligned}
$$

Let $\psi(x)=x-x_{1}-\int_{x_{1}}^{x}\left(f\left(x_{1}, v_{1}\right) / f\left(s, v_{1}\right)\right) d s$. Then, $\psi(x)$ has the global minimum at $x=x_{1}$ and $\psi\left(x_{1}\right)=0$. Furthermore, $\psi(x)>0$ for $x>0$. Hence, $V_{2}$ is positive definite with respect to $E_{1}$. For $t \geq 0$, the time derivative of $V_{2}$ along the solutions of system (2) is

$$
\begin{aligned}
& \dot{V}_{2}=\left(1-\frac{f\left(x_{1}, v_{1}\right)}{f\left(x, v_{1}\right)}\right) \dot{x}+\left(1-\frac{u_{1}}{u}\right) \dot{u} \\
& +\frac{\sigma+\mu_{2}}{\sigma}\left(1-\frac{y_{1}}{y}\right) \dot{y}+\frac{\mu_{3}\left(\sigma+\mu_{2}\right)}{k \sigma}\left(1-\frac{v_{1}}{v}\right) \dot{v} \\
& +\frac{\sigma+\mu_{2}}{\sigma} \frac{p}{q-m} \dot{z}+\frac{\sigma+\mu_{2}}{\sigma} \\
& \frac{q}{q-m}[p y(t) z(t)-p y(t-\tau) z(t-\tau)] \\
& =\left(1-\frac{f\left(x_{1}, v_{1}\right)}{f\left(x, v_{1}\right)}\right)\left(\lambda-f(x, v) v-\mu_{1} x\right) \\
& +\left(1-\frac{u_{1}}{u}\right)\left(f(x, v) v-\left(\sigma+\mu_{2}\right) u\right) \\
& +\frac{\sigma+\mu_{2}}{\sigma}\left(1-\frac{y_{1}}{y}\right)\left(\sigma u-p y z-\mu_{3} y\right) \\
& +\frac{\mu_{3}\left(\sigma+\mu_{2}\right)}{k \sigma}\left(1-\frac{v_{1}}{v}\right)\left(k y-\mu_{4} v\right)+\frac{\sigma+\mu_{2}}{\sigma} \\
& \frac{p}{q-m}\left(q y(t-\tau) z(t-\tau)-\mu_{5} z-m y z\right) \\
& +\frac{\sigma+\mu_{2}}{\sigma} \\
& \cdot \frac{q}{q-m}[p y(t) z(t)-p y(t-\tau) z(t-\tau)] \text {. }
\end{aligned}
$$

Note that $\lambda=f\left(x_{1}, v_{1}\right) v_{1}+\mu_{1} x_{1}, f\left(x_{1}, v_{1}\right) v_{1}=\left(\sigma+\mu_{2}\right) u_{1}$, and $\mu_{3} y_{1}=\sigma u_{1}$; we have

$$
\begin{aligned}
\dot{V}_{2} & =\left(1-\frac{f\left(x_{1}, v_{1}\right)}{f\left(x, v_{1}\right)}\right)\left(f\left(x_{1}, v_{1}\right) v_{1}+\mu_{1} x_{1}\right. \\
& \left.-f(x, v) v-\mu_{1} x\right)+\left(1-\frac{u_{1}}{u}\right)(f(x, v) v \\
& -\frac{\left.f\left(x_{1}, v_{1}\right) v_{1} u\right)+\frac{\sigma+\mu_{2}}{\sigma}\left(1-\frac{y_{1}}{y}\right)(-p y z)}{u_{1}}+f\left(x_{1}, v_{1}\right) v_{1}\left(1-\frac{y_{1}}{y}\right)\left(\frac{u}{u_{1}}-\frac{y}{y_{1}}\right) \\
& +\frac{\mu_{3}\left(\sigma+\mu_{2}\right)}{k \sigma}\left(1-\frac{v_{1}}{v}\right)\left(k y-\mu_{4} v\right)+\frac{\sigma+\mu_{2}}{\sigma} \\
& +\frac{p}{q-m}\left[q y(t-\tau) z(t-\tau)-\mu_{5} z\right. \\
& -m y z]+\frac{\sigma+\mu_{2}}{\sigma} \frac{q}{q-m}[p y(t) z(t) \\
& -p y(t-\tau) z(t-\tau)]=\mu_{1}\left(1-\frac{f\left(x_{1}, v_{1}\right)}{f\left(x, v_{1}\right)}\right)\left(x_{1}\right.
\end{aligned}
$$




$$
\begin{aligned}
& -x)+\frac{\sigma+\mu_{2}}{\sigma} p z\left(y_{1}-\frac{\mu_{5}}{q-m}\right)+f\left(x_{1}, v_{1}\right) v_{1}(5 \\
& -\frac{y_{1}}{y} \frac{u}{u_{1}}-\frac{f(x, v) v}{f\left(x_{1}, v_{1}\right) v_{1}} \frac{u_{1}}{u}-\frac{y}{y_{1}} \frac{v_{1}}{v}-\frac{f\left(x_{1}, v_{1}\right)}{f\left(x, v_{1}\right)} \\
& \left.-\frac{f\left(x, v_{1}\right)}{f(x, v)}\right)+f\left(x_{1}, v_{1}\right) v_{1}\left(-1-\frac{v}{v_{1}}\right. \\
& \left.+\frac{f(x, v) v}{f\left(x, v_{1}\right) v_{1}}+\frac{f\left(x, v_{1}\right)}{f(x, v)}\right) .
\end{aligned}
$$

Since the arithmetic mean is greater than or equal to the geometric mean, it has

$$
\begin{gathered}
5-\frac{y_{1}}{y} \frac{u}{u_{1}}-\frac{f(x, v) v}{f\left(x_{1}, v_{1}\right) v_{1}} \frac{u_{1}}{u}-\frac{y}{y_{1}} \frac{v_{1}}{v}-\frac{f\left(x_{1}, v_{1}\right)}{f\left(x, v_{1}\right)} \\
-\frac{f\left(x, v_{1}\right)}{f(x, v)} \leq 0 .
\end{gathered}
$$

From hypotheses (H3)-(H4), we have

$$
\begin{aligned}
-1 & -\frac{v}{v_{1}}+\frac{f(x, v) v}{f\left(x, v_{1}\right) v_{1}}+\frac{f\left(x, v_{1}\right)}{f(x, v)} \\
= & \frac{f(x, v)-f\left(x, v_{1}\right)}{f\left(x, v_{1}\right)} \frac{f(x, v) v-f\left(x, v_{1}\right) v_{1}}{f(x, v) v_{1}} \leq 0 .
\end{aligned}
$$

Note Remark 3, we have $y_{1} \leq \mu_{5} /(q-m)$. Therefore, $\dot{V}_{2} \leq$ 0 if $R_{1} \leq 1$. Then it follows from stability theorems in $[34,35]$ that the infected equilibrium without immunity $E_{1}$ is stable for any time delay $\tau \geq 0$ if $R_{1} \leq 1$.

Furthermore, note that, for each $t \geq 0, \dot{V}_{2}=0$ implies that $x(t)=x_{1}, u(t)=u_{1}, y(t)=y_{1}$, and $v(t)=v_{1}$. Let $M$ be the largest invariant set in the set

$$
\begin{aligned}
\Gamma_{2}= & \left\{\left(\varphi_{1}, \varphi_{2}, \varphi_{3}, \varphi_{4}, \varphi_{5}\right) \in C \mid \dot{V}_{2}=0\right\} \\
& \subset\left\{\left(\varphi_{1}, \varphi_{2}, \varphi_{3}, \varphi_{4}, \varphi_{5}\right) \in C \mid \varphi_{1}(0)=x_{1}, \varphi_{2}(0)\right. \\
& \left.=u_{1}, \varphi_{3}(0)=y_{1}, \varphi_{4}(0)=v_{1}\right\} .
\end{aligned}
$$

We have from system (2) and the invariance of $M$ that $M=$ $\left\{E_{1}\right\}$. Since any solution of system (2) is bounded, it follows from LaSalle invariance principle (see, e.g., $[34,35]$ ) that the infected equilibrium without immunity $E_{1}$ is also globally attractive for any time delay $\tau \geq 0$ if $R_{1} \leq 1$.

The characteristic equation of system (2) at $E_{1}$ takes the form

$$
\left(s+\mu_{5}+m y_{1}-q y_{1} e^{-s \tau}\right) \psi_{0}(s)=0,
$$

where $\psi_{0}(s)$ is a polynomial with respect to $s$. Let

$$
\psi_{1}(s)=s+\mu_{5}+m y_{1}-q y_{1} e^{-s \tau} .
$$

Thus we have $\lim _{s \rightarrow+\infty} \psi_{1}(s)>0$ and $\psi_{1}(0)=\mu_{5}-(q-m) y_{1}$. From Remark 3, we have that $(q-m) y_{1} / \mu_{5}>1$ if $R_{1}>1$. Thus, $\psi_{1}(0)<0$ if $R_{1}>1$. Hence, if $R_{1}>1$, then $\psi_{1}(s)=0$ has at least a positive real root; that is, $(38)$ has at least a positive real root. Therefore, the infected equilibrium without immunity $E_{1}$ is unstable.

\section{The Local Stability of the Infected Equilibrium and Hopf Bifurcation}

The characteristic equation of system (2) at the infected equilibrium with CTL response $E^{*}$ is given by

$$
\begin{aligned}
s^{5}+ & A_{1} s^{4}+A_{2} s^{3}+A_{3} s^{2}+A_{4} s+A_{5} \\
& +e^{-s \tau}\left(B_{1} s^{4}+B_{2} s^{3}+B_{3} s^{2}+B_{4} s+B_{5}\right)=0,
\end{aligned}
$$

where

$$
\begin{aligned}
& A_{1}=A+D+E+\mu_{4}+q y^{*}, \\
& A_{2}=\left(E+\mu_{4}\right) q y^{*}+\mu_{4} E+D\left(E+\mu_{4}+q y^{*}\right)+A(D \\
& \left.+E+\mu_{4}+q y^{*}\right)-p y^{*} m z^{*}, \\
& A_{3}=\mu_{4} E q y^{*}+D\left(\mu_{4}+E\right) q y^{*}-\sigma k B \\
& +A\left[\left(\mu_{4}+E\right) q y^{*}+\mu_{4} E+D\left(E+\mu_{4}+q y^{*}\right)\right] \\
& -p y^{*} m z^{*}\left(A+D+\mu_{4}\right) \text {, } \\
& A_{4}=-B k \sigma q y^{*}+A\left[\mu_{4} E q y^{*}+D\left(\mu_{4}+E\right) q y^{*}\right. \\
& -B k \sigma]+C k \sigma(B+H)-p y^{*} m z^{*}\left(D \mu_{4}+A \mu_{4}\right. \\
& +A D) \text {, } \\
& A_{5}=C \sigma k(B+H) q y^{*}-p y^{*} m z^{*} A D \mu_{4}-A B \sigma k q y^{*}, \\
& B_{1}=-q y^{*} \\
& B_{2}=-q y^{*}\left(A+D+\mu_{3}+\mu_{4}\right) \text {, } \\
& B_{3}=-q y^{*}\left[\mu_{3} \mu_{4}+D\left(\mu_{3}+\mu_{4}\right)+A\left(D+\mu_{3}+\mu_{4}\right)\right] \text {, } \\
& B_{4}=-q y^{*}\left[D \mu_{3} \mu_{4}+A\left(\mu_{3} \mu_{4}+D \mu_{3}+D \mu_{4}\right)\right. \\
& -\sigma k(B+H)] \text {, } \\
& B_{5}=-q y^{*}\left[A D \mu_{3} \mu_{4}-A \sigma k(B+H)+C \sigma k(B+H)\right] \text {, } \\
& a=\frac{\partial f\left(x^{*}, v^{*}\right)}{\partial x}>0 \text {, } \\
& d=\frac{\partial f\left(x^{*}, v^{*}\right)}{\partial v} \leq 0, \\
& A=a v^{*}+\mu_{1}, \\
& B=d v^{*}, \\
& C=a v^{*}, \\
& D=\sigma+\mu_{2}, \\
& E=\mu_{3}+p z^{*},
\end{aligned}
$$




$$
\begin{aligned}
& F=(q-m) z^{*}, \\
& H=f\left(x^{*}, v^{*}\right), \\
& M=p y^{*} F .
\end{aligned}
$$

When $\tau=0$, (40) becomes

$$
s^{5}+\alpha_{1} s^{4}+\alpha_{2} s^{3}+\alpha_{3} s^{2}+\alpha_{4} s+\alpha_{5}=0,
$$

where

$$
\begin{aligned}
\alpha_{1} & =A_{1}+B_{1}=A+D+E+\mu_{4}>0, \\
\alpha_{2} & =A_{2}+B_{2} \\
& =M+A\left(D+E+\mu_{4}\right)+\left(E \mu_{4}+D \mu_{4}+D E\right)>0, \\
\alpha_{3} & =A_{3}+B_{3} \\
& =\left(A+D+\mu_{4}\right) M+A\left(E \mu_{4}+D \mu_{4}+D E\right)-B k \sigma \\
& >0, \\
\alpha_{4} & =A_{4}+B_{4}=\left(D \mu_{4}+A \mu_{4}+A D\right) M+G>0, \\
\alpha_{5} & =A_{5}+B_{5}=A D \mu_{4} M>0, \\
G & =C D E \mu_{4}-\mu_{1} B k \sigma>0 .
\end{aligned}
$$

Denote

$$
\begin{aligned}
& \Delta_{1}=\alpha_{1}, \\
& \Delta_{2}=\alpha_{1} \alpha_{2}-\alpha_{3}, \\
& \Delta_{3}=\alpha_{3} \Delta_{2}+\alpha_{1} \alpha_{5}-\alpha_{1}^{2} \alpha_{4}, \\
& \Delta_{4}=\alpha_{4}\left(\Delta_{3}+\alpha_{1} \alpha_{5}\right)-\alpha_{2} \alpha_{5} \Delta_{2}-\alpha_{5}^{2}, \\
& \Delta_{5}=\alpha_{5} \Delta_{4} .
\end{aligned}
$$

Since $\mu_{4}\left(\sigma+\mu_{2}\right)\left(\mu_{3}+p z^{*}\right)=\sigma k f\left(x^{*}, v^{*}\right)$ and (H4), we have $\sigma k H=\mu_{4} D E, \mu_{4} D E+B k \sigma>0$, and $A D E \mu_{4}-G>0$.

Thus,

$$
\begin{aligned}
\Delta_{1} & =\alpha_{1}>0, \\
\Delta_{2} & =E M+A^{2}\left(D+E+\mu_{4}\right)+\left(D+E+\mu_{4}\right)[A(D \\
& \left.\left.+E+\mu_{4}\right)+D\left(E+\mu_{4}\right)+E \mu_{4}\right]+B k \sigma>0, \\
\Delta_{3} & =M\left[A \left(A^{2} E+A E^{2}+E^{2} \mu_{4}+D E \mu_{4}+E M\right.\right. \\
& +B k \sigma)+D\left(A^{2} E+D^{2} E+A D E+A E^{2}+D E^{2}\right. \\
& \left.+D E \mu_{4}+E M+B k \sigma\right)+\mu_{4}\left(A^{2} E+D^{2} E+D E \mu_{4}\right. \\
& +A D E+A E^{2}+D E^{2}+D E \mu_{4}+E^{2} \mu_{4}+E \mu_{4}^{2} \\
& +E M+B k \sigma)]+\mu_{1}\left(D E \mu_{4}+B k \sigma\right)(A+D+E
\end{aligned}
$$

$$
\begin{aligned}
& \left.+\mu_{4}\right)^{2}+A D E\left(A^{2} D+A^{2} E+A D^{2}+A D E+D^{2} E\right. \\
& \left.+D E \mu_{4}+A D E+A E^{2}+D E^{2}+E M+B k \sigma\right) \\
& +A D \mu_{4}\left(A^{2} D+A^{2} E+A^{2} \mu_{4}+A D^{2}+A D E\right. \\
& +A D \mu_{4}+D^{2} E+D^{2} \mu_{4}+A D E+A E^{2}+A E \mu_{4} \\
& +D E^{2}+D E \mu_{4}+A D \mu_{4}+A \mu_{4}^{2}+D E \mu_{4}+D \mu_{4}^{2} \\
& +E M+B k \sigma)+\left(A E \mu_{4}-B k \sigma\right) \Delta_{2}>0
\end{aligned}
$$$$
\Delta_{4}=N_{1} M^{3}+N_{2} M^{2}+N_{3} M+N_{4} \text {, }
$$

where

$$
\begin{aligned}
& N_{1}=E\left[A^{2}\left(D+\mu_{4}\right)+\left(D+\mu_{4}\right)\left(A \mu_{4}+D \mu_{4}+A D\right)\right] \\
& >0 \text {, } \\
& N_{2}=A^{2} D\left(A^{2} E+A E^{2}\right)+A^{2} \mu_{4}\left(A^{2} E+A E^{2}\right) \\
& +D^{2} \mu_{4}\left(D^{2} E+D E^{2}+D E \mu_{4}+B k \sigma\right) \\
& +A D \mu_{4}\left(A^{2} E+D^{2} E+D E^{2}\right)+A D^{2}\left(A^{2} E+D^{2} E\right. \\
& +A D E+A E^{2}+D E^{2}+D E \mu_{4}+E^{2} \mu_{4}+D E \mu_{4} \\
& +B k \sigma)+D \mu_{4}^{2}\left(D^{2} E+D E^{2}+D E \mu_{4}+E^{2} \mu_{4}\right. \\
& \left.+D E \mu_{4}+E \mu_{4}^{2}+B k \sigma\right)+\left(A \mu_{4}^{2}+a d \mu_{4}\right)\left(A^{2} E\right. \\
& +D^{2} E+A E^{2}+A D E+D E^{2}+D E \mu_{4}+E^{2} \mu_{4} \\
& \left.+A E \mu_{4}+D E \mu_{4}+E \mu_{4}^{2}+B k \sigma\right)+G E(A+D \\
& \left.+\mu_{4}\right)+A^{2} E^{2}\left(D^{2}+D \mu_{4}+\mu_{4}^{2}\right)-B k \sigma E(A D \\
& \left.+A \mu_{4}+D \mu_{4}\right)+A\left(A D+A \mu_{4}+D \mu_{4}\right)\left(D E \mu_{4}\right. \\
& +B k \sigma)>0 \text {, } \\
& N_{3}=\left[A^{2} E^{2}+A^{3} E+D^{3} E+A D E^{2}+D^{2} E^{2}+A E^{2} \mu_{4}\right. \\
& \left.+E^{2} \mu_{4}^{2}+E \mu_{4}^{3}+\left(A+D+\mu_{4}\right) B k \sigma-E B k \sigma\right] \\
& -B k \sigma\left(A D+A \mu_{4}+D \mu_{4}\right)\left(\Delta_{2}-E M\right)+\left(A^{2} E \mu_{4}^{2}\right. \\
& \left.+A^{2} D^{2} E\right)\left(A D E+D E \mu_{4}+D^{2} E+A D E+A E^{2}\right. \\
& +A E \mu_{4}+D E \mu_{4}+D E^{2}+A D \mu_{4}+A E \mu_{4}+E \mu_{4}^{2} \\
& \left.+D E \mu_{4}+A^{2} D+A^{2} E+A^{2} \mu_{4}+B k \sigma\right) \\
& +A^{2} D E \mu_{4}\left(A D E+D E \mu_{4}+A E^{2}+A E \mu_{4}+A^{2} E\right. \\
& +B k \sigma)+A^{3} E \mu_{4}^{4}+A^{2} D^{2} E\left(A D^{2}+E^{2} \mu_{4}\right) \\
& -G\left(2 D^{2} \mu_{4}^{2}+2 A D^{2} \mu_{4}+2 A D \mu_{4}^{2}+D^{3} \mu_{4}+D \mu_{4}^{3}\right),
\end{aligned}
$$




$$
\begin{aligned}
N_{4} & =G\left(A E \mu_{4}-B k \sigma\right)\left[\left(D+E+\mu_{4}\right)\right. \\
& \cdot\left(A D+A E+A \mu_{4}+E \mu_{4}+D \mu_{4}+D E\right) \\
& \left.+A^{2}\left(D+E+\mu_{4}\right)+B k \sigma\right]+G A D E\left(A D^{2}+A D E\right. \\
& +D E \mu_{4}+D^{2} E+A D E+A E^{2}+D E^{2}+A^{2} D \\
& \left.+A^{2} E+B k \sigma\right)+G A D \mu_{4}\left(A D^{2}+A D E+D E \mu_{4}\right. \\
& +D^{2} E+A D E+A E^{2}+D E^{2}+A^{2} D+A^{2} E \\
& +A D \mu_{4}+D^{2} \mu_{4}+A E \mu_{4}+A D \mu_{4}+A \mu_{4}^{2}+D \mu_{4}^{2} \\
& \left.+D E \mu_{4}+A^{2} \mu_{4}+B k \sigma\right)>0 .
\end{aligned}
$$

Assume further that

(H8) $E \geq \max \left\{\mu_{4}, D\right\}$; that is, $\mu_{3}+p z^{*} \geq \mu_{4}$ and $\mu_{3}+p z^{*} \geq$ $\sigma+\mu_{2}$.

We have

$$
\begin{aligned}
N_{3} & \geq G\left(A^{2} E^{2}+A^{3} E-E B k \sigma\right)-B k \sigma\left(A \mu_{4}+D \mu_{4}\right) \\
& \cdot\left(\Delta_{2}-E M\right)-B k \sigma A D\left(\Delta_{2}-A E \mu_{4}\right. \\
& \left.-D E \mu_{4}-E \mu_{4}^{2}\right)-B k \sigma\left(A D E \mu_{4}-G\right)\left(A+D+\mu_{4}\right) \\
& +\left(A^{2} E \mu_{4}^{2}+A^{2} D^{2} E\right)\left(A D E+D E \mu_{4}+D^{2} E\right. \\
& +A D E+A E^{2}+A E \mu_{4}+D E \mu_{4}+D E^{2}+A D \mu_{4} \\
& +A E \mu_{4}+E \mu_{4}^{2}+D E \mu_{4}+A^{2} D+A^{2} E+A^{2} \mu_{4} \\
& +B k \sigma)+A^{2} D E \mu_{4}\left(A D E+D E \mu_{4}+A E^{2}+A E \mu_{4}\right. \\
& \left.+A^{2} E+B k \sigma\right)+A^{3} E \mu_{4}^{4}+A^{2} D^{2} E\left(A D^{2}+E^{2} \mu_{4}\right) \\
& >0 .
\end{aligned}
$$

Therefore, $\Delta_{4}>0, \Delta_{5}>0$. By Routh-Hurwitz criterion, all the roots of (42) have negative real parts. Hence we have the following result.

Proposition 6. When $\tau=0$, if $\bar{R}>1, R_{1}>1$, and (H8) hold, then the infected equilibrium with CTL response $E^{*}$ is locally asymptotically stable.

In fact, when $\tau=0$, we can show that if $\bar{R}>1$ and $R_{1}>1$ hold, the infected equilibrium with CTL response $E^{*}$ is globally asymptotically stable by constructing suitable Lyapunov function.

Proposition 7. Suppose that hypotheses (H1)-(H7) and $\bar{R}>1$ are satisfied. If $R_{1}>1$, then the infected equilibrium with CTL response $E^{*}$ is globally asymptotically stable when $\tau=0$.
Proof. By the following Lyapunov function,

$$
\begin{aligned}
V_{3}= & x-x^{*}-\int_{x^{*}}^{x} \frac{f\left(x^{*}, v^{*}\right)}{f\left(s, v^{*}\right)} d s \\
& +\left(u-u^{*}-u^{*} \ln \frac{u}{u^{*}}\right) \\
& +\frac{\sigma+\mu_{2}}{\sigma}\left(y-y^{*}-y^{*} \ln \frac{y}{y^{*}}\right) \\
& +\frac{f\left(x^{*}, v^{*}\right)}{\mu_{4}}\left(v-v^{*}-v^{*} \ln \frac{v}{v^{*}}\right) \\
& +\frac{\sigma+\mu_{2}}{\sigma} \frac{p}{q-m}\left(z-z^{*}-z^{*} \ln \frac{z}{z^{*}}\right),
\end{aligned}
$$

$V_{3}$ is positive definite with respect to $E^{*}$. For $t \geq 0$, the time derivative of $V_{3}$ along the solutions of system (2) is

$$
\begin{aligned}
\dot{V}_{3}= & \left(1-\frac{f\left(x^{*}, v^{*}\right)}{f\left(x, v^{*}\right)}\right) \dot{x}+\left(1-\frac{u^{*}}{u}\right) \dot{u} \\
& +\frac{\sigma+\mu_{2}}{\sigma}\left(1-\frac{y^{*}}{y}\right) \dot{y}+\frac{f\left(x^{*}, v^{*}\right)}{\mu_{4}}\left(1-\frac{v^{*}}{v}\right) \dot{v} \\
& +\frac{\sigma+\mu_{2}}{\sigma} \frac{p}{q-m}\left(1-\frac{z^{*}}{z}\right) \dot{z} \\
= & \left(1-\frac{f\left(x^{*}, v^{*}\right)}{f\left(x, v^{*}\right)}\right)\left(\lambda-f(x, v) v-\mu_{1} x\right) \\
& +\left(1-\frac{u^{*}}{u}\right)\left(f(x, v) v-\left(\sigma+\mu_{2}\right) u\right) \\
& +\frac{\sigma+\mu_{2}}{\sigma}\left(1-\frac{y^{*}}{y}\right)\left(\sigma u-p y z-\mu_{3} y\right) \\
& +\frac{f\left(x^{*}, v^{*}\right)}{\mu_{4}}\left(1-\frac{v^{*}}{v}\right)\left(k y-\mu_{4} v\right) \\
& +\frac{\sigma+\mu_{2}}{\sigma} \frac{p}{q-m}\left(1-\frac{z^{*}}{z}\right)\left(q y z-\mu_{5} z-m y z\right) .
\end{aligned}
$$

Note that $\lambda=f\left(x^{*}, v^{*}\right) v^{*}+\mu_{1} x^{*}, f\left(x^{*}, v^{*}\right) v^{*}=\left(\sigma+\mu_{2}\right) u^{*}$, and $\mu_{3} y^{*}=\sigma u^{*}-p y^{*} z^{*}$; we have

$$
\begin{aligned}
\dot{V}_{3} & =\left(1-\frac{f\left(x^{*}, v^{*}\right)}{f\left(x, v^{*}\right)}\right)\left(f\left(x^{*}, v^{*}\right) v^{*}+\mu_{1} x^{*}\right. \\
& \left.-f(x, v) v-\mu_{1} x\right)+\left(1-\frac{u^{*}}{u}\right)(f(x, v) v \\
& \left.-\frac{f\left(x^{*}, v^{*}\right) v^{*}}{u^{*}} u\right)+\frac{\sigma+\mu_{2}}{\sigma}\left(1-\frac{y^{*}}{y}\right)\left(p y z^{*}\right. \\
& -p y z)+\frac{f\left(x^{*}, v^{*}\right) v^{*}}{\sigma u^{*}}\left(1-\frac{y^{*}}{y}\right)\left(\sigma u-\sigma u^{*} \frac{y}{y^{*}}\right) \\
& +\frac{f\left(x^{*}, v^{*}\right)}{\mu_{4}}\left(1-\frac{v^{*}}{v}\right)\left(k y-\mu_{4} v\right)+\frac{\sigma+\mu_{2}}{\sigma}
\end{aligned}
$$




$$
\begin{aligned}
& \cdot \frac{p}{q-m}\left(1-\frac{z^{*}}{z}\right)\left(q y z-\mu_{5} z-m y z\right) \\
& =\mu_{1}\left(x^{*}-x\right)\left(1-\frac{f\left(x^{*}, v^{*}\right)}{f\left(x, v^{*}\right)}\right)+f\left(x^{*}, v^{*}\right) \\
& \cdot v^{*}(-1 \\
& \left.+\frac{f(x, v)}{f\left(x, v^{*}\right)} \frac{v}{v^{*}}-\frac{v}{v^{*}}+\frac{f\left(x, v^{*}\right)}{f(x, v)}\right)+f\left(x^{*}, v^{*}\right) \\
& \cdot v^{*}(5 \\
& -\frac{f\left(x *, v^{*}\right)}{f\left(x, v^{*}\right)}-\frac{f(x, v)}{f\left(x^{*}, v^{*}\right)} \frac{v}{v^{*}} \frac{u^{*}}{u}-\frac{y^{*}}{y} \frac{u}{u^{*}} \\
& \left.-\frac{f\left(x, v^{*}\right)}{f(x, v)}-\frac{y}{y^{*}} \frac{v^{*}}{v}\right) .
\end{aligned}
$$

Since the arithmetic mean is greater than or equal to the geometric mean, it has

$$
\begin{gathered}
5-\frac{f\left(x^{*}, v^{*}\right)}{f\left(x, v^{*}\right)}-\frac{f(x, v)}{f\left(x^{*}, v^{*}\right)} \frac{v}{v^{*}} \frac{u^{*}}{u}-\frac{y^{*}}{y} \frac{u}{u^{*}} \\
-\frac{f\left(x, v^{*}\right)}{f(x, v)}-\frac{y}{y^{*}} \frac{v^{*}}{v} \leq 0 .
\end{gathered}
$$

From hypotheses (H3)-(H4), we have

$$
\begin{aligned}
-1 & +\frac{f(x, v)}{f\left(x, v^{*}\right)} \frac{v}{v^{*}}-\frac{v}{v^{*}}+\frac{f\left(x, v^{*}\right)}{f(x, v)} \\
& =\frac{f(x, v)-f\left(x, v^{*}\right)}{f\left(x, v^{*}\right)} \frac{f(x, v) v-f\left(x, v^{*}\right) v^{*}}{f(x, v) v^{*}} \leq 0 .
\end{aligned}
$$

Therefore, $\dot{V}_{3} \leq 0$ if $R_{1}>1$. Then it follows from stability theorems in $[34,35]$ that the infected equilibrium CTL response $E^{*}$ is stable for $\tau=0$ if $R_{1}>1$. Similarly, by LaSalle invariance principle, we can show that the infected equilibrium CTL response $E^{*}$ is also globally attractive for $\tau=0$ if $R_{1}>1$.

Next, we consider the case when $\tau>0$. Since $\alpha_{5}>0, s=0$ is not a root of (40). We suppose (40) has a purely imaginary root $s=i \omega(\omega>0)$ for some $\tau>0$. Substituting $s=i \omega$ into (40) and separating the real and imaginary parts, we have

$$
\begin{aligned}
\omega^{5}-A_{2} \omega^{3}+A_{4} \omega= & \left(B_{1} \omega^{4}-B_{3} \omega^{2}+B_{5}\right) \sin \omega \tau \\
& +\left(B_{2} \omega^{3}-B_{4} \omega\right) \cos \omega \tau, \\
A_{1} \omega^{4}-A_{3} \omega^{2}+A_{5}= & -\left(B_{1} \omega^{4}-B_{3} \omega^{2}+B_{5}\right) \cos \omega \tau \\
& +\left(B_{2} \omega^{3}-B_{4} \omega\right) \sin \omega \tau .
\end{aligned}
$$

Squaring and adding the two equations of (53), it follows that

$$
\omega^{10}+C_{1} \omega^{8}+C_{2} \omega^{6}+C_{3} \omega^{4}+C_{4} \omega^{2}+C_{5}=0
$$

where

$$
\begin{aligned}
& C_{1}=A_{1}^{2}-2 A_{2}-B_{1}^{2}, \\
& C_{2}=A_{2}^{2}+2 A_{4}-2 A_{1} A_{3}+2 B_{1} B_{3}-B_{2}^{2}, \\
& C_{3}=A_{3}^{2}-2 A_{2} A_{4}-B_{3}^{2}+2 B_{2} B_{4}+2 A_{1} A_{5}-2 B_{1} B_{5}, \\
& C_{4}=A_{4}^{2}-B_{4}^{2}-2 A_{3} A_{5}+2 B_{3} B_{5}, \\
& C_{5}=A_{5}^{2}-B_{5}^{2} .
\end{aligned}
$$

Letting $v=\omega^{2},(54)$ can be written as

$$
h(\nu)=v^{5}+C_{1} v^{4}+C_{2} v^{3}+C_{3} v^{2}+C_{4} v+C_{5}=0 .
$$

Then we have

$$
h^{\prime}(\nu)=5 v^{4}+4 C_{1} v^{3}+3 C_{2} v^{2}+2 C_{3} v+C_{4} .
$$

Denote

$$
\begin{aligned}
& p_{1}=-\frac{6}{25} C_{1}^{2}+\frac{3}{5} C_{2}, \\
& q_{1}=\frac{8}{125} C_{1}^{3}-\frac{6}{25} C_{1} C_{2}+\frac{2}{5} C_{3}, \\
& r_{1}=-\frac{3}{625} C_{1}^{4}+\frac{3}{125} C_{1}^{2} C_{2}-\frac{2}{25} C_{1} C_{3}+\frac{1}{5} C_{4}, \\
& \Theta_{0}=p_{1}^{2}-4 r_{1}, \\
& p_{2}=-\frac{1}{3} p_{1}^{2}-4 r_{1}, \\
& q_{2}=-\frac{2}{27} p_{1}^{3}+\frac{8}{3} p_{1} r_{1}-q_{1}^{2}, \\
& \Theta_{1}=\frac{1}{27} p_{2}^{3}+\frac{1}{4} q_{2}^{2}, \\
& s_{*}=\sqrt[3]{-\frac{q_{2}}{2}+\sqrt{\Theta_{1}}+\sqrt[3]{-\frac{q_{2}}{2}}-\sqrt{\Theta_{1}}+\frac{1}{3} p_{1},} \\
& \Theta_{3}=-s_{*}-p_{1}-\frac{2 q_{1}}{\sqrt{s_{*}-p_{1}}} .
\end{aligned}
$$

By a similar argument as that in [38], we have the following results.

Lemma 8. For the polynomial equation (56), the following results hold. 
(i) Equation (56) has at least one positive root, if one of the following conditions $(a)-(d)$ holds:

(a) $C_{5}<0$.

(b) $C_{5} \geq 0, q_{1}=0, \Theta_{0} \geq 0$, and $p_{1}<0$ or $r_{1} \leq 0$ and there exists $v^{*} \in\left\{v_{1}, v_{2}, v_{3}, v_{4}\right\}$ such that $v^{*}>$ 0 and $h\left(\nu^{*}\right) \leq 0$, where $\nu_{i}=y_{i}-(1 / 5) C_{1}(i=$ $1,2,3,4)$, and

$$
\begin{aligned}
& y_{1}=\sqrt{\frac{-p_{1}+\sqrt{\Theta_{0}}}{2}}, \\
& y_{2}=-\sqrt{\frac{-p_{1}+\sqrt{\Theta_{0}}}{2}}, \\
& y_{3}=\sqrt{\frac{-p_{1}-\sqrt{\Theta_{0}}}{2}}, \\
& y_{4}=-\sqrt{\frac{-p_{1}-\sqrt{\Theta_{0}}}{2}} .
\end{aligned}
$$

(c) $C_{5} \geq 0, q_{1} \neq 0, s_{*}>p_{1}, \Theta_{2} \geq 0$, or $\Theta_{3} \geq 0$ and there exists $v^{*} \in\left\{v_{1}^{*}, v_{2}^{*}, v_{3}^{*}, v_{4}^{*}\right\}$ such that $v^{*}>0$ and $h\left(v^{*}\right) \leq 0$, where $v_{i}=y_{i}-(1 / 5) C_{1}(i=$ $1,2,3,4)$, and

$$
\begin{aligned}
& y_{1}=\frac{-\sqrt{s_{*}-p_{1}}+\sqrt{\Theta_{2}}}{2}, \\
& y_{2}=\frac{-\sqrt{s_{*}-p_{1}}-\sqrt{\Theta_{2}}}{2}, \\
& y_{3}=\frac{\sqrt{s_{*}-p_{1}}+\sqrt{\Theta_{3}}}{2}, \\
& y_{4}=\frac{\sqrt{s_{*}-p_{1}}-\sqrt{\Theta_{3}}}{2} .
\end{aligned}
$$

(d) $C_{5} \geq 0, q \neq 0, s_{*}<p_{1}, q_{1}^{2} / 4\left(p_{1}-s_{*}\right)^{2}+$ $(1 / 2) s_{*}=0, \bar{v}>0$, and $h(\bar{v}) \leq 0$, where $\bar{v}=q_{1} / 2\left(p_{1}-s_{*}\right)-(1 / 5) C_{1}$.

(ii) If the conditions (a)-(d) of (i) are all not satisfied, then (56) has no positive real root.

Suppose that $h(\nu)=0$ has positive real roots. Without loss of generality, we may assume that (56) has $\bar{k}(1 \leq \bar{k} \leq$ 5) positive real roots, denoted, respectively, as $v_{1}, v_{2}, \ldots, v_{\bar{k}}$. Then, (54) has positive real roots $\omega_{\bar{k}}=\sqrt{v_{\bar{k}}}$. From (40), we get

$$
\cos \omega \tau=\frac{\left(\omega^{5}-A_{2} \omega^{3}+A_{4} \omega\right)\left(B_{2} \omega^{3}-B_{4} \omega\right)-\left(A_{1} \omega^{4}-A_{3} \omega^{2}+A_{5}\right)\left(B_{1} \omega^{4}-B_{3} \omega^{2}+B_{5}\right)}{\left(B_{2} \omega^{3}-B_{4} \omega\right)^{2}+\left(B_{1} \omega^{4}-B_{3} \omega^{2}+B_{5}\right)^{2}} \equiv L(\omega) .
$$

Therefore, let

$$
\tau_{k}^{(j)}=\frac{1}{\omega_{k}}\left\{\arccos L\left(\omega_{k}\right)+2 \pi j\right\}
$$

where $k=1,2, \ldots, \bar{k}, j=0,1, \ldots$. Then $\pm i \omega_{k}$ are a pair of purely imaginary roots of (54) with $\tau=\tau_{k}^{(j)}$.

Define

$$
\begin{aligned}
\tau_{0} & =\tau_{k 0}^{(0)}=\min _{k \in\{1,2, \ldots, \bar{k}\}}\left\{\tau_{k}^{(0)}\right\}, \\
\omega_{0} & =\omega_{k 0} .
\end{aligned}
$$

Let $s(\tau)=\xi(\tau)+i \omega(\tau)$ be a root of (40) satisfying $\xi\left(\tau_{k}^{(j)}\right)=0$ and $\omega\left(\tau_{k}^{(j)}\right)=\omega_{k}$. Differentiating the two sides of (40) with respect to $\tau$ and noticing that $s$ is a function of $\tau$, it follows that

$$
\begin{aligned}
\left(\frac{d s}{d \tau}\right)^{-1} & \\
= & -\frac{5 s^{4}+4 A_{1} s^{3}+3 A_{2} s^{2}+2 A_{3} s+A_{4}}{s\left(s^{5}+A_{1} s^{4}+A_{2} s^{3}+A_{3} s^{2}+A_{4} s+A_{5}\right)} \\
& +\frac{4 B_{1} s^{3}+3 B_{2} s^{2}+2 B_{3} s+B_{4}}{s\left(B_{1} s^{4}+B_{2} s^{3}+B_{3} s^{2}+B_{4} s+B_{5}\right)}-\frac{\tau}{s} .
\end{aligned}
$$

Thus, we get

$$
\begin{aligned}
{\left[\frac{d(\operatorname{Res}(\tau))}{d \tau}\right]_{\tau=\tau_{k}^{(j)}}^{-1}=} & -\frac{\left(5 \omega_{k}^{4}-3 A_{2} \omega_{k}^{2}+A_{4}\right)\left(-\omega_{k}^{6}+A_{2} \omega_{k}^{4}-A_{4} \omega_{k}^{2}\right)-\left(4 A_{1} \omega_{k}^{3}-2 A_{3} \omega_{k}\right)\left(A_{1} \omega_{k}^{5}-A_{3} \omega_{k}^{3}+A_{5} \omega_{k}\right)}{\left(-\omega_{k}^{6}+A_{2} \omega_{k}^{4}-A_{4} \omega_{k}^{2}\right)^{2}+\left(A_{1} \omega_{k}^{5}-A_{3} \omega_{k}^{3}+A_{5} \omega_{k}\right)^{2}} \\
& +\frac{\left(-3 B_{2} \omega_{k}^{2}+B_{4}\right)\left(B_{2} \omega_{k}^{4}-B_{4} \omega_{k}^{2}\right)+\left(-4 B_{1} \omega_{k}^{3}+2 B_{3} \omega_{k}\right)\left(B_{1} \omega_{k}^{5}-B_{3} \omega_{k}^{3}+B_{5} \omega_{k}\right)}{\left(B_{2} \omega_{k}^{4}-B_{4} \omega_{k}^{2}\right)^{2}+\left(B_{1} \omega_{k}^{5}-B_{3} \omega_{k}^{3}+B_{5} \omega_{k}\right)^{2}} .
\end{aligned}
$$


From (40), we attain

$$
\begin{gathered}
\left(\omega^{5}-A_{2} \omega^{3}+A_{4} \omega\right)^{2}+\left(A_{1} \omega^{4}-A_{3} \omega^{2}+A_{5}\right)^{2} \\
=\left(B_{2} \omega^{3}-B_{4} \omega\right)^{2}+\left(B_{1} \omega^{4}-B_{3} \omega^{2}+B_{5}\right)^{2} .
\end{gathered}
$$

Then

$$
\begin{aligned}
& {\left[\frac{d(\operatorname{Res}(\tau))}{d \tau}\right]_{\tau=\tau_{k}^{(j)}}^{-1}} \\
& \quad=\frac{5 v_{k}^{4}+4 C_{1} v_{k}^{3}+3 C_{2} v_{k}^{2}+2 C_{3} \nu_{k}+C_{4}}{\left(B_{1} \omega_{k}^{4}-B_{3} \omega_{k}^{2}+B_{5}\right)^{2}+\left(B_{2} \omega_{k}^{2}-B_{4}\right)^{2} \omega_{k}^{2}} \\
& =\frac{h^{\prime}\left(v_{k}\right)}{\left(B_{1} \omega_{k}^{4}-B_{3} \omega_{k}^{2}+B_{5}\right)^{2}+\left(B_{2} \omega_{k}^{2}-B_{4}\right)^{2} \omega_{k}^{2}} .
\end{aligned}
$$

Therefore, it follows that

$$
\begin{aligned}
& \operatorname{sign}\left[\frac{d(\operatorname{Res}(\tau))}{d \tau}\right]_{\tau=\tau_{k}^{(j)}}=\operatorname{sign}\left[\frac{d(\operatorname{Res}(\tau))}{d \tau}\right]_{\tau=\tau_{k}^{(j)}}^{-1} \\
& =\operatorname{sign}\left[h^{\prime}\left(v_{k}\right)\right]
\end{aligned}
$$

Since $v_{k}>0$, we can know that $\operatorname{Re}\left[d s_{k}(\tau) /\left.d \tau\right|_{\tau}=\tau_{k}^{(j)}\right]$ and $h^{\prime}\left(v_{k}\right)$ have the same sign.

From the above analysis, we have the following results.

Theorem 9. Let $\tau_{k}^{(j)}, \tau_{0}$, and $\omega_{0}$ be defined by (62) and (63). If $\bar{R}>1$ and $R_{1}>1$ are satisfied, then the following results hold:

(i) If the conditions (a)-(d) of Lemma 8 are all not satisfied, then the infected equilibrium with CTL response $E^{*}$ is locally asymptotically stable for all time delay $\tau>0$.

(ii) If one of the conditions (a)-(d) of Lemma 8 is satisfied, then the infected equilibrium with CTL response $E^{*}$ is locally asymptotically stable for $\tau \in\left[0, \tau_{0}\right)$ and unstable for $\tau>\tau_{0}$.

(iii) If all the conditions as stated in (ii) hold and $h^{\prime}\left(\nu_{k}\right) \neq$ 0 , then system (2) undergoes a Hopf bifurcation at $E^{*}$ when $\tau=\tau_{k}^{(j)}(j=0,1,2, \ldots)$.

\section{Conclusion and Numerical Simulations}

In this paper, we proposed a class of delayed HIV infection model (2) with general incidence rate and immune impairment. This general incidence only satisfies some general hypotheses and includes many types of special incidence functions as special cases. First, we discussed the nonnegativity and boundedness of the solutions and the existence of equilibria of system (2). Then, by constructing suitable Lyapunov functionals and using Lyapunov-LaSalle invariance principle and Hopf bifurcation theorem, we proved the following results.

If $R_{0} \leq 1$, the infection-free equilibrium $E_{0}$ is globally asymptotically stable for any time delay $\tau \geq 0$; that is, any solution $(x(t), u(t), y(t), v(t), z(t)) \rightarrow E_{0}=\left(x_{0}, 0,0,0,0\right)$. In biology, this means that the virus can be finally cleared from the body and the disease dies out. At the same time, as the time $t$ increases, the numbers of latently infected cells, actively infected cells, and CTLs trends to zero and the number of uninfected cells trends to a constant $x_{0}$.

If $R_{0}>1 \geq R_{1}$ and $\bar{R}>1$, the infected equilibrium without immunity $E_{1}$ is globally asymptotically stable for any time delay $\tau \geq 0$; that is, any solution $(x(t), u(t), y(t), v(t), z(t)) \rightarrow$ $E_{1}=\left(x_{1}, u_{1}, y_{1}, v_{1}, 0\right)$. In biology, this indicates that the HIV infection will finally become chronic with no persistent CTL response.

If $R_{1}>1$ and $\bar{R}>1$, there exists a unique infected equilibrium with CTL response $E^{*}$. The result of Theorem 9 implies that the time delay $\tau$ can destabilize the stability of the infected equilibrium with CTL response $E^{*}$ and leads to the occurrence of Hopf bifurcations.

If the time delay $\tau \in\left[0, \tau_{0}\right)$, the infected equilibrium with CTL response $E^{*}$ is locally asymptotically stable. In biology, this implies that the HIV infection may become chronic and the CTL immune response may be persistent. When the time delay $\tau$ passes through the critical value $\tau_{0}$, the infected equilibrium with CTL response $E^{*}$ will become unstable and a Hopf bifurcation occurs under some conditions. In biology, this suggests that as the time delay $\tau$ increases, the numbers of the uninfected cells, latently infected cells, actively infected cells, free virus, and CTLs will first attend constant values and then become oscillated.

We now give numerical simulations to illustrate the main results in Sections 3 and 4.

Let us choose $f(x, v) v=\beta x v$. Then we have that $R_{0}=$ $k \lambda \beta \sigma /\left(\mu_{1} \mu_{3} \mu_{4}\left(\sigma+\mu_{2}\right)\right)$ and $R_{1}=R_{0}-k \beta \mu_{5} /\left(\mu_{1} \mu_{4}(q-m)\right)$. Based on the numerical simulations in $[12,19,21,22,27]$, let us take the following data:

$$
\begin{aligned}
\lambda & =270, \quad \beta=0.001, \quad k=6, \quad m=0.001, \\
p & =0.04, \quad q=0.025, \quad \sigma=0.001, \quad \mu_{1}=0.02, \\
\mu_{2} & =0.1, \quad \mu_{3}=0.8, \quad \mu_{4}=1.2, \quad \mu_{5}=0.05 .
\end{aligned}
$$

Direct calculations show that $R_{0}=0.8354<1$ and $R_{1}=$ $0.3146<1$; system (2) has the infection-free equilibrium $E_{0}=(13500,0,0,0,0)$. By Theorem 4, the infection-free equilibrium $E_{0}$ is globally asymptotically stable for any time delay $\tau \geq 0$. Figure 1 gives the phase trajectories of system (2) with suitable initial condition.

Next, let us choose the following data:

$$
\begin{aligned}
\lambda & =270, \quad \beta=0.001, \quad k=6, \quad m=0.01, \\
p & =0.04, \quad q=0.025, \quad \sigma=0.002, \quad \mu_{1}=0.02, \\
\mu_{2} & =0.1, \quad \mu_{3}=0.8, \quad \mu_{4}=1.2, \quad \mu_{5}=0.05 .
\end{aligned}
$$

Direct computations show that $R_{0}=1.6544>1, \bar{R}=$ $1.9853>1$, and $R_{1}=0.8211<1$; system (2) has the infected equilibrium without immunity $E_{1}=(8160,1047.0588$, $2.6176,13.0882,0)$. Therefore, by Theorem 5 , the infected equilibrium without immunity $E_{1}$ is globally asymptotically 


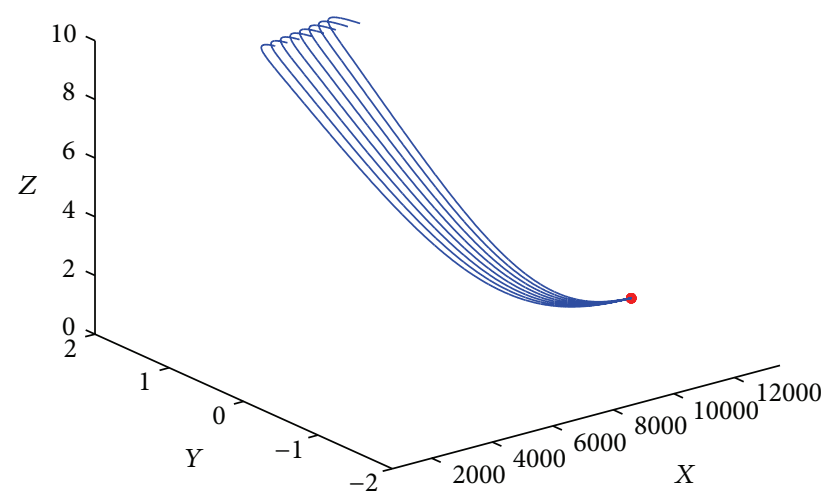

FIGURE 1: Phase trajectories of system (2) with $R_{0} \leq 1$.

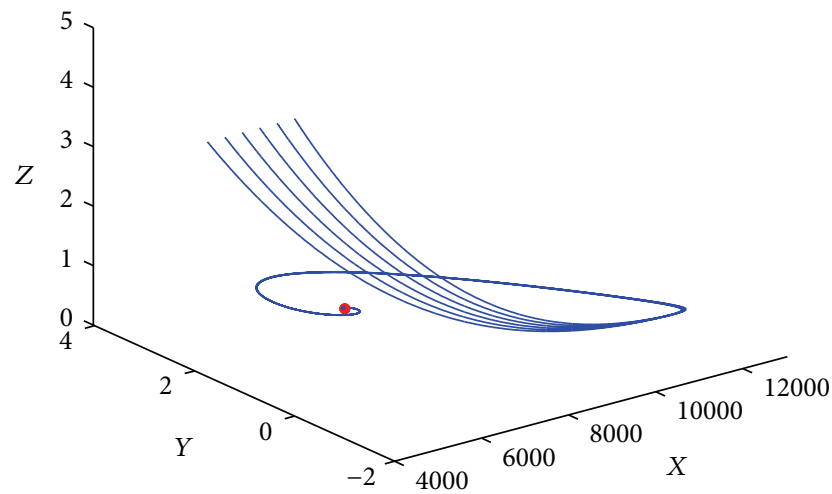

FIgURE 2: Phase trajectories of system (2) with $R_{0}>1 \geq R_{1}$.

stable for any time delay $\tau \geq 0$. Figure 2 gives the phase trajectories of system (2) with suitable initial condition.

Furthermore, let us choose the following data:

$$
\begin{aligned}
\lambda & =270, \quad \beta=0.001, \quad k=6, \quad m=0.012, \\
p & =0.04, \quad q=0.025, \quad \sigma=0.004, \quad \mu_{1}=0.02, \\
\mu_{2} & =0.1, \quad \mu_{3}=0.8, \quad \mu_{4}=1.2, \quad \mu_{5}=0.05 .
\end{aligned}
$$

Then we have that $R_{0}=3.2452>1, \bar{R}=3.375>1$, and $R_{1}=$ $2.2837>1$ and (56) has no positive root. System (2) has the infected equilibrium with CTL response $E^{*}=(6882.3529$, $1272.6244,3.84615,19.2308,13.0882)$. From Theorem 9(i), the infected equilibrium with CTL response $E^{*}$ is locally asymptotically stable for any time delay $\tau>0$. Figure 3 gives the phase trajectories of system (2) with suitable initial condition.

Finally, let us choose the following data:

$$
\begin{aligned}
\lambda & =270, \quad \beta=0.001, \quad k=6, \quad m=0.01, \\
p & =0.04, \quad q=0.025, \quad \sigma=0.004, \quad \mu_{1}=0.02, \\
\mu_{2} & =0.1, \quad \mu_{3}=0.8, \quad \mu_{4}=1.2, \quad \mu_{5}=0.05 .
\end{aligned}
$$

Then we have that $R_{0}=3.2452>1, \bar{R}=3.8942>1$, and $R_{1}=2.4119>1$, (56) has two positive roots, and $h^{\prime}\left(v_{k}\right) \neq 0$. By simple computations, we have $\omega_{0} \approx 0.0394$ and

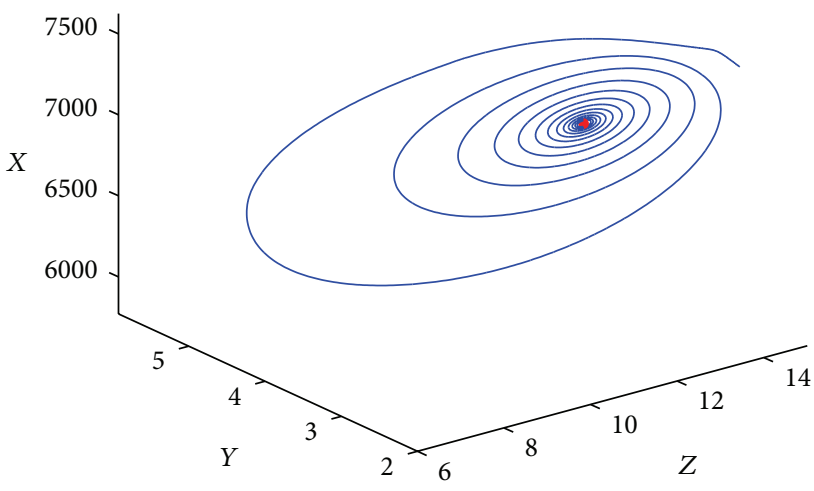

Figure 3: Phase trajectories of system (2) with $R_{1}>1, \tau=28$. The initial condition is $(7970,1000,2.6,0.2,17)$.

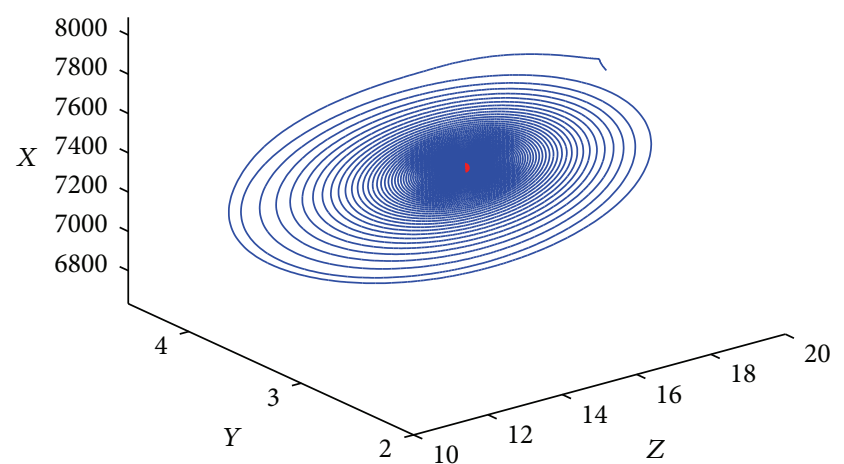

FIGURE 4: Phase trajectories of system (2) with $R_{1}>1, \tau=25<\tau_{0}$. The initial condition is $(7970,1000,2.6,0.2,17)$.

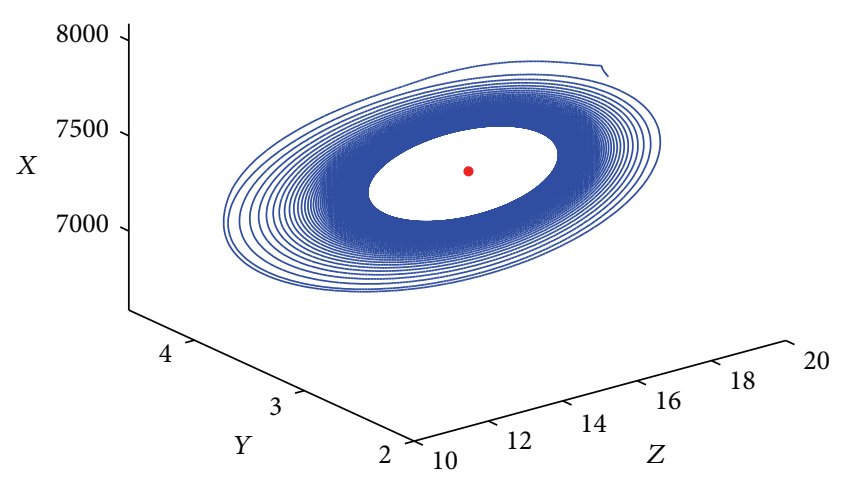

FIGURE 5: Phase trajectories of system (2) with $R_{1}>1, \tau=28>\tau_{0}$. The initial condition is $(7970,1000,2.6,0.2,17)$.

$\tau_{0} \approx 27.2546$. From Theorem 9(ii), the infected equilibrium with CTL response $E^{*}=(7363.6364,1180.0699,3.3333$, $16.6667,15.4021)$ is asymptotically stable if $0<\tau<\tau_{0}$ and unstable if $\tau>\tau_{0}$. Figure 4 gives the phase trajectories of system (2) with $\tau<\tau_{0}$ and suitable initial condition. Figure 5 gives the phase trajectories of system (2) with $\tau>\tau_{0}$ and suitable initial condition and shows the occurrence of the Hopf bifurcations.

Since $v^{*}=k \mu_{5} /\left(\mu_{4}(q-m)\right)$ and $z^{*}=\mu_{1} \mu_{3} \mu_{4}(q-m)\left(R_{1}-\right.$ 1)/ $\left(p \mu_{1} \mu_{4}(q-m)+p \beta k \mu_{5}\right)$, it is easy to see that the number of 


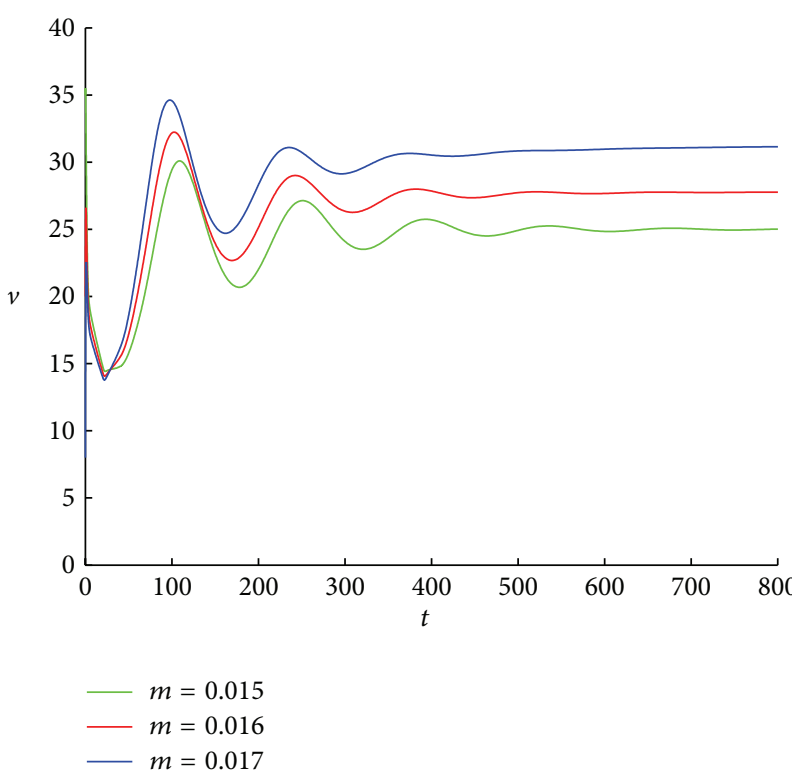

Figure 6: The curves of the free virus of system (2) with $\tau=20, m=0.015,0.016$, and 0.017 . The initial conditions are chosen as $(7000,1000,8,35,10),(7000,1000,8,20,10)$, and $(7000,1000,8,8,10)$, respectively.

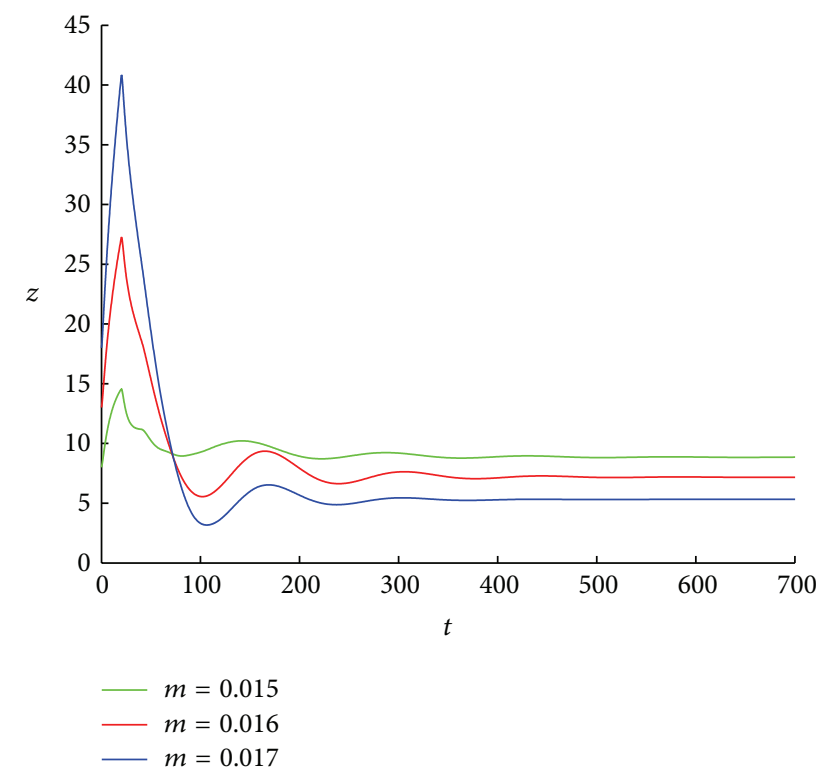

FIgURE 7: The curves of the CTLs of system (2) with $\tau=20$, $m=0.015,0.016$, and 0.017 . The initial conditions are chosen as $(7000,1000,8,2,8),(7000,1000,8,2,13)$, and $(7000,1000,8,2,18)$, respectively.

free viruses is increased and the number of CTLs is decreased with respect to the immune impairment rate $m$. For example, if we choose $m=0.015$, then $v_{1}^{*}=25$ and $z_{1}^{*}=8.8462$. If we choose $m=0.016$, then $v_{2}^{*}=27.7778$ and $z_{2}^{*}=7.1691$. If we choose $m=0.017$, then $v_{3}^{*}=31.25$ and $z_{3}^{*}=5.3283$. Figure 6 shows that, for any initial conditions, when $m=$ $0.015,0.016,0.017$, the numbers of free viruses trend to $v_{1}^{*}$, $v_{2}^{*}$, and $v_{3}^{*}$, respectively. Figure 7 shows that, for any initial conditions, when $m=0.015,0.016,0.017$, the numbers of CTLs trend to $z_{1}^{*}, z_{2}^{*}$, and $z_{3}^{*}$, respectively. In Figures 6 and 7 , all the data are chosen as in Figure 4 except the immune impairment rate $m$.

As immune impairment rate $m$ increases, the CTL response gradually becomes weak and the individuals eventually develop AIDS. Thus, in order to control the HIV infection, we should decrease the value of $m$. Numerical simulations show the similar known results (see, e.g., [22]).

\section{Conflict of Interests}

The authors declare that there is no conflict of interests regarding the publication of this paper.

\section{Acknowledgments}

The authors thank the editor and anonymous reviewers of the journal for their helpful and valuable comments. The research is supported by NNSF of China (11471034).

\section{References}

[1] M. A. Nowak and R. M. May, Virus Dynamics, Oxford University, New York, NY, USA, 2000.

[2] A. S. Perelson and P. W. Nelson, "Mathematical analysis of HIV1 dynamics in vivo," SIAM Review, vol. 41, no. 1, pp. 3-44, 1999.

[3] A. S. Perelson, A. U. Neumann, M. Markowitz, J. M. Leonard, and D. D. Ho, "HIV-1 dynamics in vivo: virion clearance rate, infected cell life-span, and viral generation time," Science, vol. 271, no. 5255, pp. 1582-1586, 1996.

[4] M. A. Nowak and C. R. M. Bangham, "Population dynamics of immune responses to persistent viruses," Science, vol. 272, no. 5258, pp. 74-79, 1996.

[5] A. A. Canabarro, I. M. Gléria, and M. L. Lyra, "Periodic solutions and chaos in a non-linear model for the delayed cellular immune response," Physica A: Statistical Mechanics and its Applications, vol. 342, no. 1-2, pp. 234-241, 2004.

[6] D. Li and W. Ma, "Asymptotic properties of a HIV-1 infection model with time delay," Journal of Mathematical Analysis and Applications, vol. 335, no. 1, pp. 683-691, 2007.

[7] Y. Xiao, H. Miao, S. Tang, and H. Wu, "Modeling antiretroviral drug responses for HIV-1 infected patients using differential equation models," Advanced Drug Delivery Reviews, vol. 65, no. 7, pp. 940-953, 2013.

[8] K. Wang, W. Wang, H. Pang, and X. Liu, "Complex dynamic behavior in a viral model with delayed immune response," Physica D: Nonlinear Phenomena, vol. 226, no. 2, pp. 197-208, 2007.

[9] T. Kajiwara and T. Sasaki, "A note on the stability analysis of pathogen-immune interaction dynamics," Discrete and Continuous Dynamical Systems, Series B, vol. 4, no. 3, pp. 615-622, 2004.

[10] W.-M. Liu, "Nonlinear oscillations in models of immune responses to persistent viruses," Theoretical Population Biology, vol. 52, no. 3, pp. 224-230, 1997.

[11] X. Wang, W. Wang, and P. Liu, "Global properties of an HIV dynamic model with latent infection and CTL immune 
responses," Journal of Southwest University for Nationalities(Natural Science Edition), vol. 35, pp. 68-72, 2013 (Chinese).

[12] R. R. Regoes, D. Wodarz, and M. A. Nowak, "(V)irus dynamics: $f$ he effect of target cell limitation and immune responses on virus evolution," Journal of Theoretical Biology, vol. 191, no. 4, pp. 451-462, 1998.

[13] N. Burić, M. Mudrinic, and N. Vasović, "Time delay in a basic model of the immune response," Chaos, Solitons and Fractals, vol. 12, no. 3, pp. 483-489, 2001.

[14] A. Korobeinikov, "Global properties of basic virus dynamics models," Bulletin of Mathematical Biology, vol. 66, no. 4, pp. 879-883, 2004.

[15] Z. Hu, J. Zhang, H. Wang, W. Ma, and F. Liao, "Dynamics analysis of a delayed viral infection model with logistic growth and immune impairment," Applied Mathematical Modelling, vol. 38, no. 2, pp. 524-534, 2014.

[16] X. Shi, X. Zhou, and X. Song, "Dynamical behavior of a delay virus dynamics model with CTL immune response," Nonlinear Analysis: Real World Applications, vol. 11, no. 3, pp. 1795-1809, 2010.

[17] C. Lv, L. Huang, and Z. Yuan, "Global stability for an HIV-1 infection model with Beddington-DeAngelis incidence rate and CTL immune response," Communications in Nonlinear Science and Numerical Simulation, vol. 19, no. 1, pp. 121-127, 2014.

[18] K. Lassen, Y. Han, Y. Zhou, J. Siliciano, and R. F. Siliciano, “The multifactorial nature of HIV-1 latency," Trends in Molecular Medicine, vol. 10, no. 11, pp. 525-531, 2004.

[19] L. Rong and A. S. Perelson, "Asymmetric division of activated latently infected cells may explain the decay kinetics of the HIV1 latent reservoir and intermittent viral blips," Mathematical Biosciences, vol. 217, no. 1, pp. 77-87, 2009.

[20] B. Buonomo and C. Vargas-De-León, "Global stability for an HIV-1 infection model including an eclipse stage of infected cells," Journal of Mathematical Analysis and Applications, vol. 385, no. 2, pp. 709-720, 2012.

[21] E. Avila-Vales, N. Chan-Chí, and G. García-Almeida, "Analysis of a viral infection model with immune impairment, intracellular delay and general non-linear incidence rate," Chaos, Solitons \& Fractals, vol. 69, pp. 1-9, 2014.

[22] S. Wang, X. Song, and Z. Ge, "Dynamics analysis of a delayed viral infection model with immune impairment," Applied Mathematical Modelling, vol. 35, no. 10, pp. 4877-4885, 2011.

[23] E. S. Rosenberg, M. Altfeld, S. H. Poon et al., "Immune control of HIV-1 after early treatment of acute infection," Nature, vol. 407, no. 6803, pp. 523-526, 2000.

[24] N. L. Komarova, E. Barnes, P. Klenerman, and D. Wodarz, "Boosting immunity by antiviral drug therapy: ${ }^{s}$ simple relationship among timing, efficacy, and success," Proceedings of the National Academy of Sciences of the United States of America, vol. 100, no. 4, pp. 1855-1860, 2003.

[25] S. A. Kalams and B. D. Walker, "The critical need for CD4 help in maintaining effective cytotoxic T lymphocyte responses," The Journal of Experimental Medicine, vol. 188, no. 12, pp. 21992204, 1998.

[26] S. Iwami, T. Miura, S. Nakaoka, and Y. Takeuchi, "Immune impairment in HIV infection: existence of risky and immunodeficiency thresholds," Journal of Theoretical Biology, vol. 260, no. 4, pp. 490-501, 2009.

[27] Z. Wang and X. Liu, "A chronic viral infection model with immune impairment," Journal of Theoretical Biology, vol. 249, no. 3, pp. 532-542, 2007.
[28] G. Huang, Y. Takeuchi, and W. Ma, "Lyapunov functionals for delay differential equations model of viral infections," SIAM Journal on Applied Mathematics, vol. 70, no. 7, pp. 2693-2708, 2010.

[29] K. Hattaf, N. Yousfi, and A. Tridane, "Mathematical analysis of a virus dynamics model with general incidence rate and cure rate," Nonlinear Analysis: Real World Applications, vol. 13, no. 4, pp. 1866-1872, 2012.

[30] T. Wang, Z. Hu, F. Liao, and W. Ma, "Global stability analysis for delayed virus infection model with general incidence rate and humoral immunity," Mathematics and Computers in Simulation, vol. 89, pp. 13-22, 2013.

[31] K. Hattaf, A. A. Lashari, Y. Louartassi, and N. Yousfi, "A delayed SIR epidemic model with general incidence rate," Electronic Journal of Qualitative Theory of Differential Equations, vol. 3, pp. $1-9,2013$

[32] P. H. Crowley and E. K. Martin, "Functional responses and interference within and between year classes of a dragonfly population," Journal of the North American Benthological Society, vol. 8, no. 3, pp. 211-221, 1989.

[33] X. Song and A. U. Neumann, "Global stability and periodic solution of the viral dynamics," Journal of Mathematical Analysis and Applications, vol. 329, no. 1, pp. 281-297, 2007.

[34] Y. Kuang, Delay Differential Equations with Applications in Population Dynamics, Academic Press, Boston, Mass, USA, 1993.

[35] J. K. Hale and S. M. Verduyn Lunel, Introduction to Functional Differential Equations, Springer, NewYork, NY, USA, 1993.

[36] A. Korobeinikov, "Global properties of infectious disease models with nonlinear incidence," Bulletin of Mathematical Biology, vol. 69, no. 6, pp. 1871-1886, 2007.

[37] C. C. McCluskey, "Lyapunov functions for tuberculosis models with fast and slow progression," Mathematical Biosciences and Engineering, vol. 3, no. 4, pp. 603-614, 2006.

[38] Y. Yang and J. Ye, "Stability and bifurcation in a simplified fiveneuron BAM neural network with delays," Chaos, Solitons and Fractals, vol. 42, no. 4, pp. 2357-2363, 2009. 


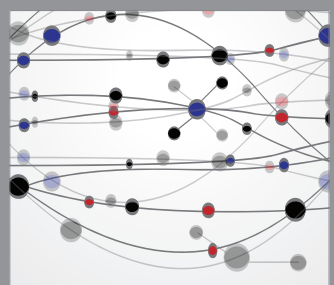

The Scientific World Journal
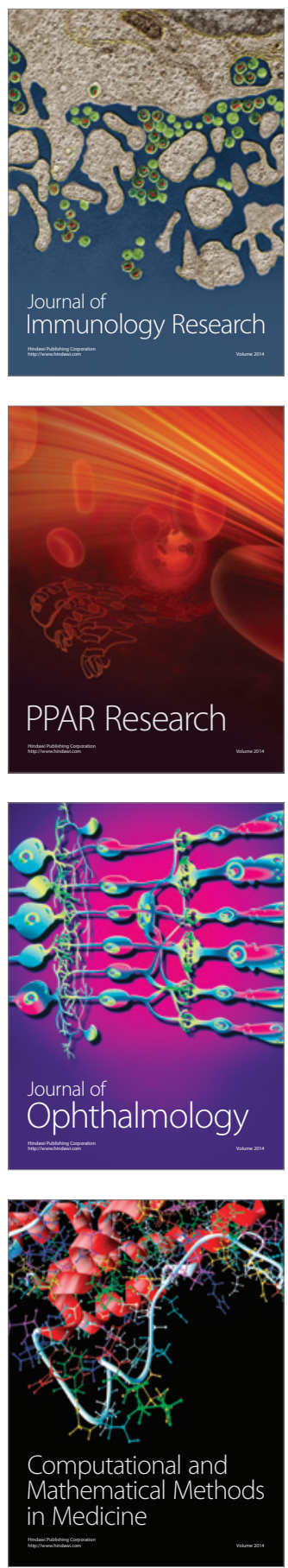

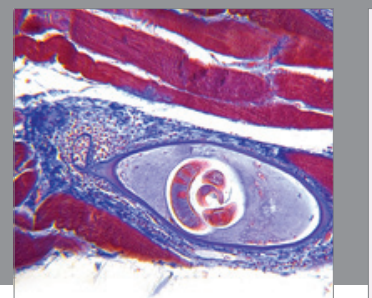

Gastroenterology

Research and Practice
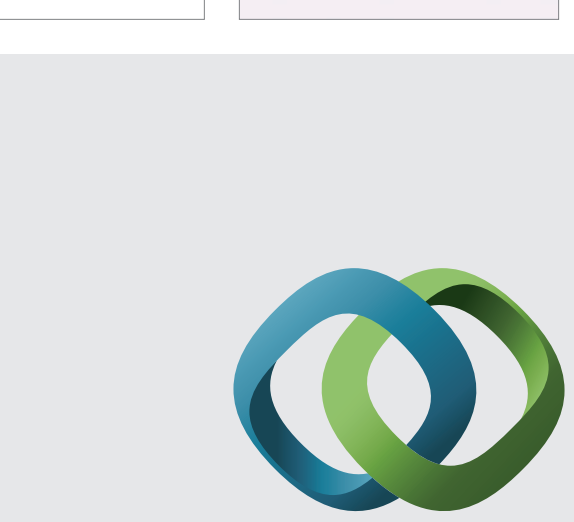

\section{Hindawi}

Submit your manuscripts at

http://www.hindawi.com
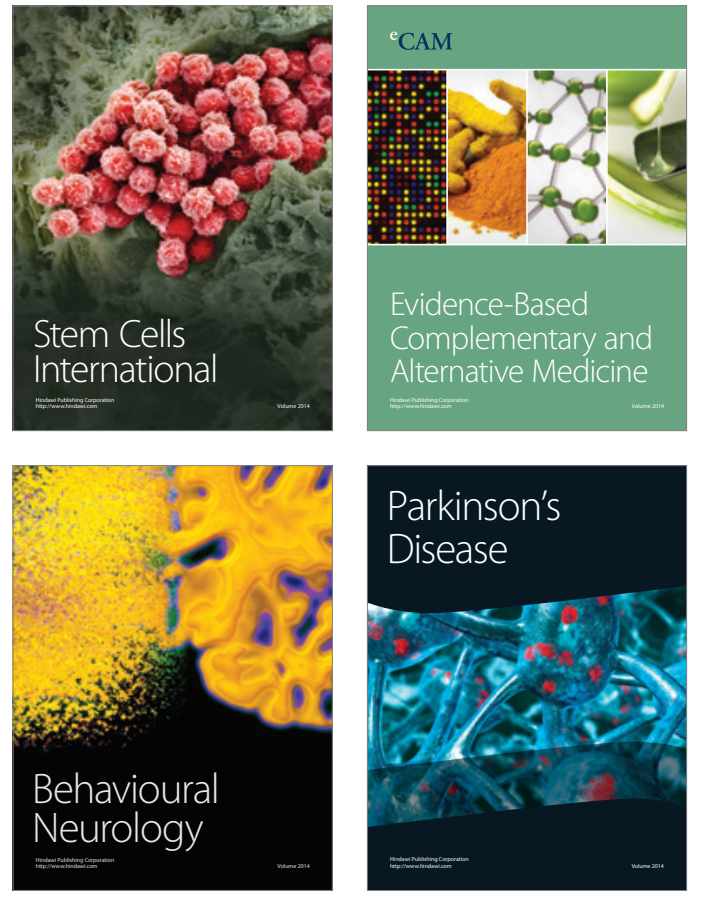
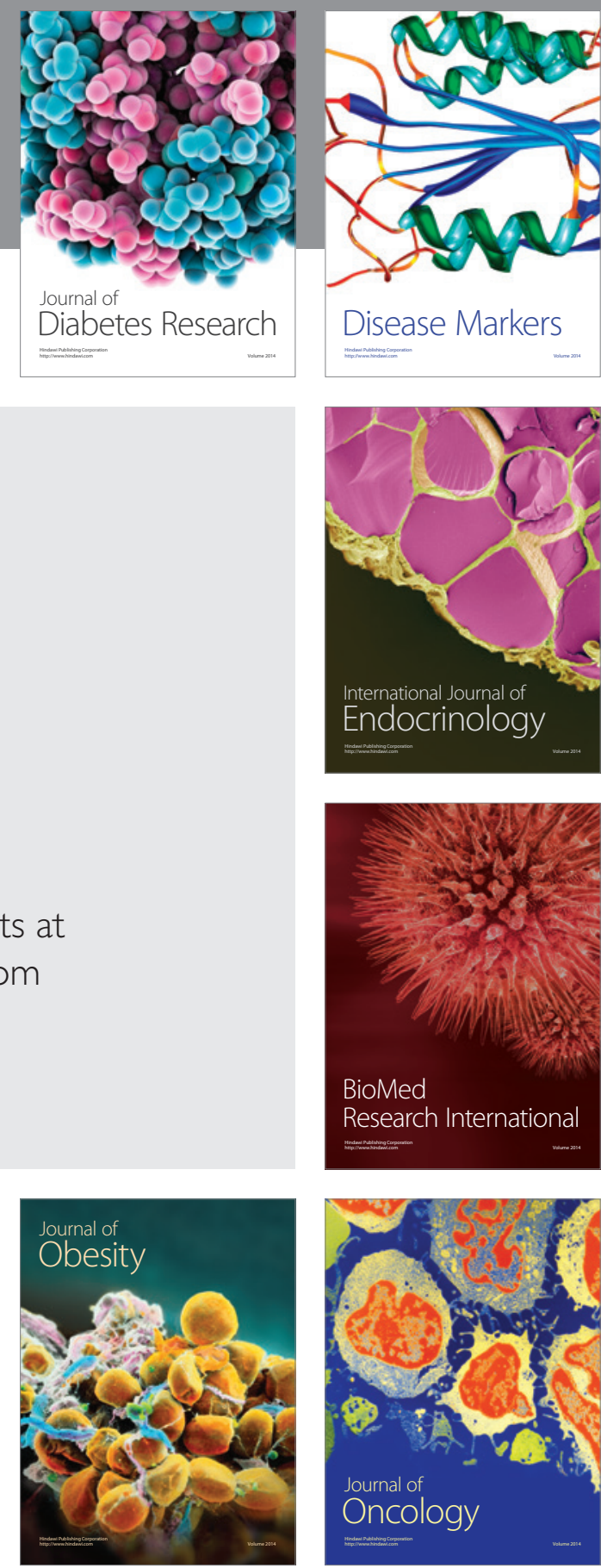

Disease Markers
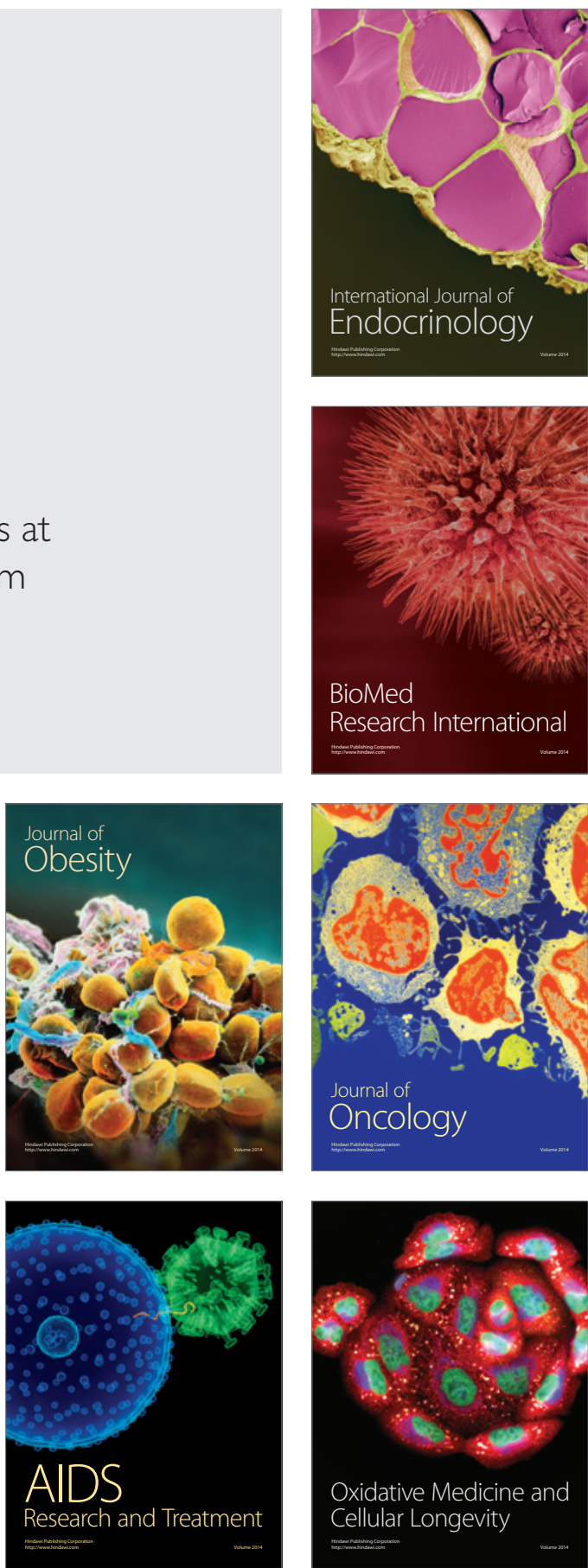